\title{
A cooperative coevolutionary algorithm for the Multi-Depot Vehicle Routing Problem
}

\author{
Fernando Bernardes de Oliveira ${ }^{\mathrm{a}, \mathrm{c}}$, Rasul Enayatifar ${ }^{\mathrm{a}}$, Hossein Javedani Sadaei ${ }^{\mathrm{a}}$, \\ Frederico Gadelha Guimarães ${ }^{\mathrm{b}, *}$, Jean-Yves Potvin ${ }^{\mathrm{d}}$ \\ ${ }^{a}$ Graduate Program in Electrical Engineering, Federal University of Minas Gerais, Av. Antônio Carlos 6627, 31270-901 Belo Horizonte, MG, Brazil \\ ${ }^{\mathrm{b}}$ Department of Electrical Engineering, Universidade Federal de Minas Gerais, UFMG, Belo Horizonte, Brazil \\ ${ }^{\mathrm{c}}$ Departamento de Computação e Sistemas, Universidade Federal de Ouro Preto, UFOP, João Monlevade, MG, Brazil \\ ${ }^{\mathrm{d}}$ Centre interuniversitaire de recherche sur les réseaux d'entreprise, la logistique et le transport (CIRRELT), Université de Montréal, C.P. 6128, succursale \\ Centre-ville, Montréal, Québec, Canada H3C 3J7
}

\section{A R T I C L E I N F O}

\section{Keywords:}

Multi-Depot Vehicle Routing Problem

Vehicle routing

Cooperative coevolutionary algorithm

Evolution strategies

\begin{abstract}
A B S T R A C T
The Multi-Depot Vehicle Routing Problem (MDVRP) is an important variant of the classical Vehicle Routing Problem (VRP), where the customers can be served from a number of depots. This paper introduces a cooperative coevolutionary algorithm to minimize the total route cost of the MDVRP. Coevolutionary algorithms are inspired by the simultaneous evolution process involving two or more species. In this approach, the problem is decomposed into smaller subproblems and individuals from different populations are combined to create a complete solution to the original problem. This paper presents a problem decomposition approach for the MDVRP in which each subproblem becomes a single depot VRP and evolves independently in its domain space. Customers are distributed among the depots based on their distance from the depots and their distance from their closest neighbor. A population is associated with each depot where the individuals represent partial solutions to the problem, that is, sets of routes over customers assigned to the corresponding depot. The fitness of a partial solution depends on its ability to cooperate with partial solutions from other populations to form a complete solution to the MDVRP. As the problem is decomposed and each part evolves separately, this approach is strongly suitable to parallel environments. Therefore, a parallel evolution strategy environment with a variable length genotype coupled with local search operators is proposed. A large number of experiments have been conducted to assess the performance of this approach. The results suggest that the proposed coevolutionary algorithm in a parallel environment is able to produce high-quality solutions to the MDVRP in low computational time.
\end{abstract}

(c) 2015 Elsevier Ltd. All rights reserved.

\section{Introduction}

A Vehicle Routing Problem (VRP) is a generic name for a large class of combinatorial optimization problems (Doerner \& Schmid, 2010; Montoya-Torres, Franco, Isaza, Jiménez, \& Herazo-Padilla, 2015). The goal is to find a set of routes for serving customers with a certain number of vehicles in a given environment. In the classical VRP, a problem instance is specified by a set of customers to be served with their corresponding locations and demands and other primary information such as distance between two costumers, distance between a customer and the depot, number of vehicles and vehicle capacity

\footnotetext{
* Corresponding author. Tel.: +55 3134093419.

E-mail addresses: fboliveira25@gmail.com (F.B. de Oliveira), r.enayatifar@gmail.com (R. Enayatifar), hjavedani@gmail.com (H.J. Sadaei), fredericoguimaraes@ufmg.br, frederico.g.guimaraes@gmail.com (F.G. Guimarães), potvin@iro.umontreal.ca (J.-Y. Potvin).
}

(Baldacci \& Mingozzi, 2009). In a solution, each vehicle leaves the depot and executes a route over a certain number of customers before returning to the depot, while insuring that the total demand on the route does not exceed vehicle capacity. In some cases, a maximum route duration (or distance) constraint is enforced. The Multi-Depot Vehicle Routing Problem (MDVRP) is a variant of the classical VRP in which more than one depot is considered (Cordeau \& Maischberger, 2012; Escobar, Linfati, Toth, \& Baldoquin, 2014; Subramanian, Uchoa, \& Ochi, 2013; Vidal, Crainic, Gendreau, Lahrichi, \& Rei, 2012).

The number of studies on the MDVRP is rather limited when compared to the classical VRP. A survey of these studies, based on either exact methods or heuristics, can be found in Montoya-Torres et al. (2015). In recent years, evolutionary-based metaheuristics proved to be a popular approach to address this problem, as described in Section 3. But, in spite of this popularity, no coevolutionary algorithm has yet been proposed in the literature for the MDVRP. As the problem can be easily decomposed into a number of single-depot VRPs, with a 


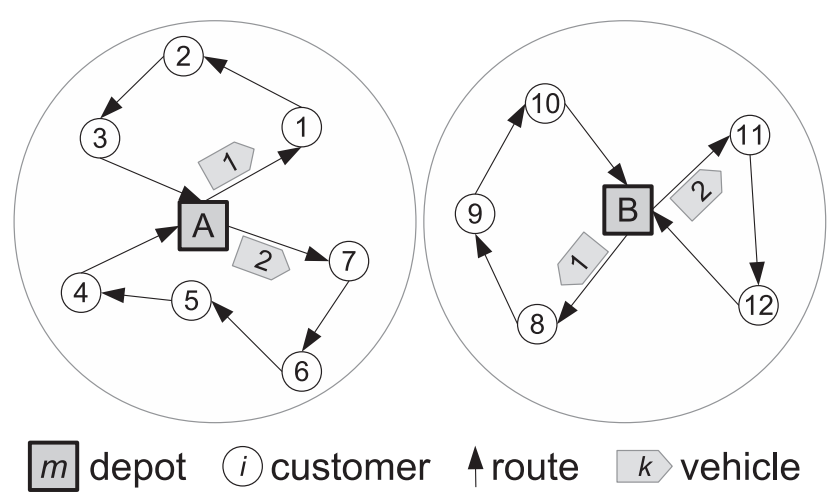

Fig. 1. MDVRP solution.

population of partial solutions associated with each depot, a coevolutionary approach looks relevant. Each partial solution or individual in a population corresponds to vehicle routes defined over the subset of customers assigned to the corresponding depot. Although each population can evolve separately, this evolution is guided by the ability of each individual to form good complete solutions with individuals from the other populations. This is the problem-solving approach proposed in this work.

The remainder of this paper is organized as follows. First, some preliminaries about the MDVRP and coevolution are found in Section 2. Section 3 presents a literature review. Sections 4 and 5 describe the proposed methodology while Section 6 reports computational results. Future avenues for research are proposed in the conclusion in Section 7.

\section{Preliminaries}

In this section, some preliminary information about the mathematical formulation of the MDVRP and cooperative coevolutionary algorithms are presented.

\subsection{Multi-Depot Vehicle Routing Problem formulation}

As mentioned earlier, the MDVRP is a variant of the classical VRP where more than one depot is considered (Montoya-Torres et al., 2015). Fig. 1 shows a typical solution of this problem with two depots and two vehicle routes associated with each depot. Typically, the fleet of vehicles is limited and homogeneous (Cordeau \& Maischberger, 2012; Escobar et al., 2014; Montoya-Torres et al., 2015; Subramanian et al., 2013; Vidal et al., 2012).

Basically, a solution to this problem is a set of vehicle routes such that: (i) each vehicle route starts and ends at the same depot, (ii) each customer is served exactly once by one vehicle, (iii) the total demand on each route does not exceed vehicle capacity (iv) the maximum route time is satisfied and (v) the total cost is minimized (Montoya-Torres et al., 2015).

The MDVRP can be formalized as follows. Let $G=(V, A)$ be a complete graph, where $V$ is the set of nodes and $A$ is the set of arcs. The nodes are partitioned into two subsets: the customers to be served, $V_{C}=\{1, \ldots, N\}$, and the multiple depots $V_{D}=\{N+1, \ldots, N+M\}$, with $V_{C} \cup V_{D}=V$ and $V_{C} \cap V_{D}=\oslash$. There is a non-negative cost $c_{i j}$ associated with each $\operatorname{arc}(i, j) \in A$. The demand of each customer is $d_{i}$ (there is no demand at the depot nodes). There is also a fleet of $K$ identical vehicles, each with capacity $Q$. The service time at each customer $i$ is $t_{i}$ while the maximum route duration time is set to $T$. A conversion factor $w_{i j}$ might be needed to transform the cost $c_{i j}$ into time units. In this work, however, the cost is the same as the time and distance units, so $w_{i j}=1$.

In the mathematical formulation that follows, binary variables $x_{i j k}$ are equal to 1 when vehicle $k$ visits node $j$ immediately after node $i$.
Auxiliary variables $y_{i}$ are also used in the subtour elimination constraints.

Minimize $\sum_{i=1}^{N+M} \sum_{j=1}^{N+M} \sum_{k=1}^{K} c_{i j} x_{i j k}$

subject to:

$\sum_{i=1}^{N+M} \sum_{k=1}^{K} x_{i j k}=1(j=1, \ldots, N) ;$
$\sum_{j=1}^{N+M} \sum_{k=1}^{K} x_{i j k}=1(i=1, \ldots, N) ;$

$\sum_{i=1}^{N+M} x_{i h k}-\sum_{j=1}^{N+M} x_{h j k}=0(k=1, \ldots, K ; h=1, \ldots, N+M) ;$

$\sum_{i=1}^{N+M} \sum_{j=1}^{N+M} d_{i} x_{i j k} \leq Q(k=1, \ldots, K) ;$

$\sum_{i=1}^{N+M} \sum_{j=1}^{N+M}\left(c_{i j} w_{i j}+t_{i}\right) x_{i j k} \leq T(k=1, \ldots, K) ;$

$\sum_{i=N+1}^{N+M} \sum_{j=1}^{N} x_{i j k} \leq 1(k=1, \ldots, K) ;$

$\sum_{j=N+1}^{N+M} \sum_{i=1}^{N} x_{i j k} \leq 1(k=1, \ldots, K)$;

$y_{i}-y_{j}+(M+N) x_{i j k} \leq N+M-1$;

for $1 \leq i \neq j \leq N$ and $1 \leq k \leq K$;

$x_{i j k} \in\{0,1\} \forall i, j, k$;

$y_{i} \in\{0,1\} \forall i$

The objective (1) minimizes the total cost. Constraints (2) and (3) guarantee that each customer is served by exactly one vehicle. Flow conservation is guaranteed through constraint (4). Vehicle capacity and route duration constraints are found in (5) and (6), respectively. Constraints (7) and (8) check vehicle availability. Subtour elimination constraints are in (9). Finally, (10) and (11) define $x$ and $y$ as binary variables.

In the original formulation of the MDVRP, a fixed number of vehicles is allocated to each depot. In our work, though, the search is allowed to consider a larger number of vehicles (at a penalty cost in the objective). This is discussed in Section 5.

\subsection{Coevolutionary algorithms}

Coevolutionary algorithms are a class of evolutionary algorithms inspired by the simultaneous evolution process involving two or more species. Recently, various engineering problems have been solved with this approach (Blecic, Cecchini, \& Trunfio, 2014; Chen, Mori, \& Matsuba, 2014; Ladjici \& Boudour, 2011; Ladjici, Tiguercha, \& Boudour, 2014; Wang \& Chen, 2013a; Wang, Cheng, \& Huang, 2014). Coevolutionary algorithms are categorized into two groups depending on the type of interaction among the species, which can be either competitive or cooperative. Competitive coevolution can be viewed as an arms race, that is, individuals in the populations compete among themselves. One group attempts to take advantage over another, which responds with an adaptive strategy to recover the advantage (Katada \& Handa, 2010). A biological example is the predator-prey competitive coevolution, in which the evolution of one population 


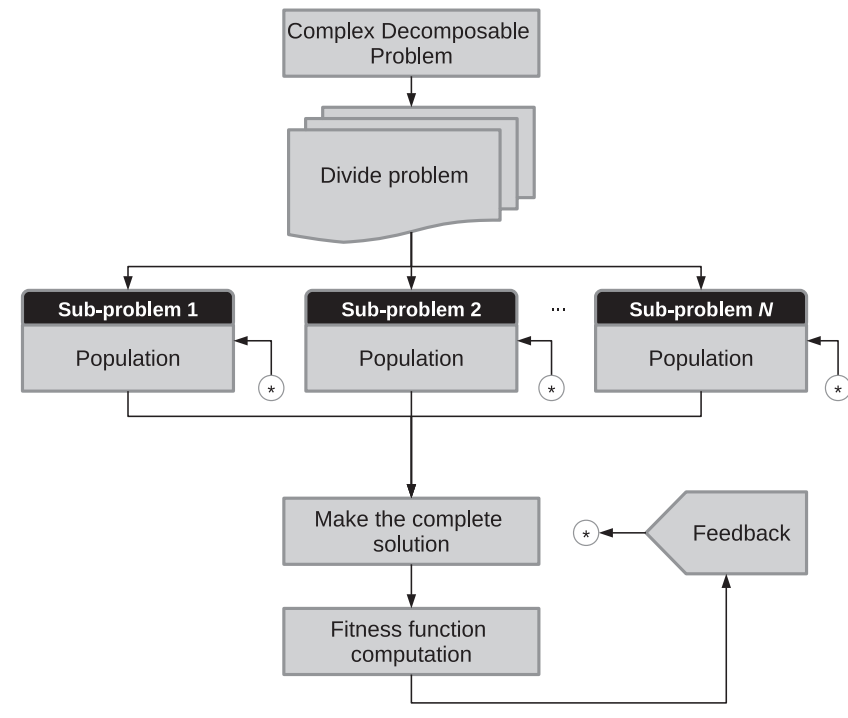

Fig. 2. Cooperative coevolutionary algorithm with problem decomposition.

affects the evolution of the other. This approach has been used in virtual and simulated evolution (Ebner, 2006) and also for evolving agent behaviors or artificial players for games (Engelbrecht, 2007). In cooperative coevolution, the interaction among species is beneficial for all or, at least, does not cause any damage. The species may live together in the same area and one population needs the other ones to survive and evolve. Typically, species cooperate to attain a global benefit. This is the case in symbiosis, for example Engelbrecht (2007).

In a cooperative coevolutionary algorithm, each population represents a part of a complex decomposable problem (Engelbrecht, 2007). Accordingly, the true fitness of an individual can only be obtained from its interaction with other individuals from the same or other populations. Each individual receives a reward or punishment, being rewarded when it interacts well with the others while getting a punishment otherwise. A cooperative coevolutionary algorithm is considered in this work because the problem can be decomposed into smaller subproblems, each one evolving in parallel. Partial solutions for those subproblems cooperate to create a complete solution for the MDVRP. In this situation, a competitive strategy would not be suitable because a complete solution is obtained from information gathered from all subproblems and there is no competition among these subproblems. Fig. 2 depicts a general cooperative coevolutionary algorithm with decomposition. A complex problem is first decomposed into smaller subproblems. Each population is evolved on its subproblem and, after a number of generations, individuals from these populations are combined to create complete solutions to the original problem. Through this process, it is possible to compute the fitness of these complete solutions. Some feedback information is then returned to each population, such as the best solution found so far, any required updates to the individuals in the population, etc.

Coevolutionary algorithms can be distinguished from traditional evolutionary algorithms by their evaluation process (Ficici, 2004). As mentioned above, individuals can only be evaluated through interaction with other individuals from the same or different populations.

Fitness sampling (Engelbrecht, 2007) or fitness assessment (Luke, 2013) defines how individuals are combined for the purpose of fitness evaluation. These methods are the followings (Engelbrecht, 2007; Luke, 2013):

(a) All versus all. All possible combinations of individuals from all populations are considered. This method is very expensive and can be appropriate for populations with only a few individuals. (b) Random. Individuals are randomly selected from the coevolving populations. This method is less expensive than the previous one and the number of evaluations is typically a parameter of the method.

(c) All versus best. All individuals of one population are combined with the best individuals from other populations. This is repeated for each population.

(d) Tournament sampling. This process consists of selecting individuals from each population based on their fitness. Then, a tournament is performed among all selected individuals to determine a winner. This is typically used in competitive models.

(e) Shared sampling. Only individuals with higher shared fitness are combined to favor individuals that are significantly different from the others in a population.

\section{Literature review}

The literature review focuses on the main issues addressed in this work. First, Section 3.1 introduces evolutionary-based algorithms for the MDVRP, followed by parallel algorithms for the MDVRP in Section 3.2. Then, Section 3.3 is devoted to known applications of sequential and parallel coevolutionary algorithms.

\subsection{Heuristics for the Multi-Depot Vehicle Routing Problem}

Evolutionary algorithms (EAs) use a set of candidate solutions, known as a population, and heuristic mechanisms to evolve it like selection and reproduction (also called genetic operators). Evolution proceeds from one generation to the next until a stopping condition is satisfied (Engelbrecht, 2007; Luke, 2013). Evolutionary algorithms have been widely used to solve the VRP, as surveyed in Potvin (2009). The main contributions with regard to the MDVRP are reported below.

Genetic algorithms (GAs) are probably the most widely used class of evolutionary algorithms and were applied as well to the MDVRP. A comprehensive survey of different types of GAs for the MDVRP can be found in Karakatič and Podgorelec (2015), while GAs for the MDVRP, among other VRP variants, are also described in Gendreau, Potvin, Bräumlaysy, Hasle, and Løkketangen (2008). With regard to hybrids, a simulated annealing-based solution acceptance criterion is applied after reproduction in Chen and Xu (2008). The Clarke and Wright savings heuristic and the nearest neighbor heuristic are used in Ho, Ho, Ji, and Lau (2008) to create initial solutions for the GAs. A combination of simulated annealing, bee colony optimization and GA is also proposed in Liu (2013). Vidal et al. (2012) and Vidal, Crainic, Gendreau, and Prins (2014) introduce a powerful hybrid GA using neighborhood-based heuristics and population-diversity management schemes to address many different types of VRPs, including the MDVRP.

Particle swarm optimization (PSO) is inspired by the social behavior of agents, such as swarms and birds flock. Individuals are named particles, flying in the search space according to simple rules that combine local and global information (Engelbrecht, 2007; Luke, 2013). This problem-solving methodology was used in Wenjing and Ye (2010) for solving the MDVRP.

In addition to EAs, some noteworthy heuristics were proposed to solve the MDVRP. Tabu Search (TS) has been used in several contexts. In particular, Renaud, Laporte, and Boctor (1996) and Cordeau, Gendreau, and Laporte (1997) use this heuristic for some VRP problems including MDVRP. A hybrid granular TS algorithm was proposed by Escobar et al. (2014). Those authors introduce the idea of granular neighborhoods, in which the search process uses restricted neighborhoods for each customer defined by a granularity threshold value.

An adaptive large neighborhood search (ALNS) approach was introduced by Pisinger and Ropke (2007). It was applied to solve five different variants of the VRP, including the MDVRP. 
The method developed by Subramanian et al. (2013) combines an exact procedure based on the set partitioning formulation with an Iterated Local Search (ILS). A Mixed Integer Programming solver is used for the exact procedure.

\subsection{Parallel algorithms for the Multi-Depot Vehicle Routing Problem}

A few parallel algorithms for the MDVRP are reported in the literature. A parallel version of Ant Colony Optimization (ACO) is introduced in Yu, Yang, and Xie (2011). In this work, the MDVRP was simplified through the definition of a single virtual depot, while insuring that the capacity constraint of each vehicle is satisfied. The algorithm was implemented using a distributed coarse-grained environment composed of eight computers each equipped with a Pentium processor (3 $\mathrm{GHz}$ with $512 \mathrm{MB}$ RAM).

A parallel iterated Tabu Search (TS) is proposed in Maischberger and Cordeau (2011) and Cordeau and Maischberger (2012). In the first paper, some preliminary results are reported on eight classes of VRPs including the MDVRP. The algorithm was run on a cluster made of 128 nodes (each with a $3 \mathrm{GHz}$ Xeon processor). The second paper reports results over four VRP variants, including the MDVRP, as well as other variants with time windows.

\subsection{Coevolutionary algorithms}

In this section, we review different applications of CAs. Note that a discussion about sequential and parallel versions of CAs can be found in Popovici and De Jong (2006). The authors show in particular how different population update strategies can impact the overall performance. The authors empirically demonstrate the superiority of the parallel version over the sequential one on benchmark functions, using a number of different metrics.

A competitive model with three populations is used in Li, Guimarães, and Lowther (2015) to solve constrained design problems. The first population is made of candidate solutions from the design space while the other populations represent disturbances due to uncertainties. Li and Yao (2012) propose a cooperative coevolutionary algorithm in a particle swarm environment. They apply it to large scale optimization problems on functions with up to 2000 variables. Other methodologies using particle swarm and coevolution can also be found in Aote, Raghuwanshi, and Malik (2015) and Chen, Zhu, and Hu (2010).

A study on sequential and parallel versions of a cooperative coevolutionary algorithms based on the ES(1+1) evolution strategy is presented in Jansen and Wiegand (2003) 2004). The authors identify situations where a cooperative scheme could be inappropriate, like problems involving non separable functions. Depending on the decomposition method or the characteristics of the function, the coevolutionary algorithm could even be harmful.

As far as we know, the coevolutionary paradigm has never been applied to the MDVRP. With regard to vehicle routing in general, a large scale capacitated arc routing problem is addressed in $\mathrm{Mei}, \mathrm{Li}$, and Yao (2014) using a coevolutionary algorithm. In this work, the routes are grouped into different subsets to be optimized and problem instances with more than 300 edges are solved. A multi-objective capacitated arc routing problem is also studied in Shang et al. (2014). A coevolutionary algorithm is presented in Wang and Chen (2013b) for a pickup and delivery problem with time windows. To minimize the number of vehicles and the total traveling distance, the authors use two populations: one for diversification purposes and the other for intensification purposes. In the scheduling domain, a competitive coevolutionary quantum genetic algorithm for minimizing the makespan of a job shop scheduling problem is reported in $\mathrm{Gu}, \mathrm{Gu}$, Cao, and Gu (2010).

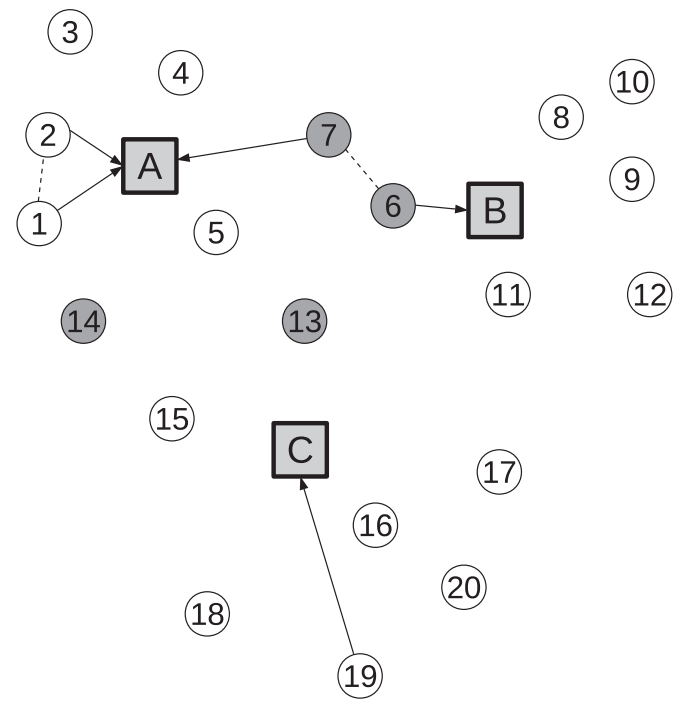

Fig. 3. Problem decomposition: assignment of customers to depots.

\section{Cooperative coevolutionary model for the MDVRP}

A cooperative coevolutionary model with problem decomposition is proposed here to solve the MDVRP. The main contribution of this method is the computational efficiency resulting from the decomposition of the problem into subproblems. Each subproblem becomes a single depot VRP and evolves independently in its domain space. The decomposition approach considers the depots separately and assigns a subset of customers to each one. Some overlap is possible, that is, customers might be associated with one or more depots depending on their neighbors (other customers or depots). An evolving population is associated with each subproblem and the (partial) solutions in each population must then cooperate to form a complete solution to the MDVRP. In this section, we explain the decomposition approach. Then, Section 5 describes the evolution process for solving the subproblems.

Each customer is assigned to a depot using the two following rules:

1. The closest depot.

2. The closest depot to the closest neighbor node (if different from the one in rule 1 ).

When the two rules identify the same depot, the assignment is obvious. When the two rules identify two different depots, then the conflict must be addressed in some way. Fig. 3 illustrates how these rules are applied. In the figure, there are three depots (nodes A-C) and 20 customers (nodes 1-20). For customer 1, rule 1 identifies $A$ as the closest depot. Then, rule 2 identifies the closest neighbor to customer 1 as customer 2, whose closest depot is also A. Thus, customer 1 can only be assigned to depot A. In the figure, all white nodes can only be assigned to their closest depot.

Now, let us consider customer 6. Rule 1 identifies $B$ as the closest depot. Then, rule 2 identifies the closest neighbor to customer 6 as customer 7, whose closest depot is A. Since we do not know if it is better to assign customer 6 to depot A or B, customer 6 is initially assigned to both depots. That is, this customer will be part of the two evolving populations associated with depots $A$ and $B$. It implies that there is some overlap among the subproblems. In Fig. 3, all gray nodes are assigned to two different depots.

The assignment procedure is further detailed in Algorithm 1 . The procedure starts with the set of customers $V_{C}$, the set of depots $V_{D}$, 

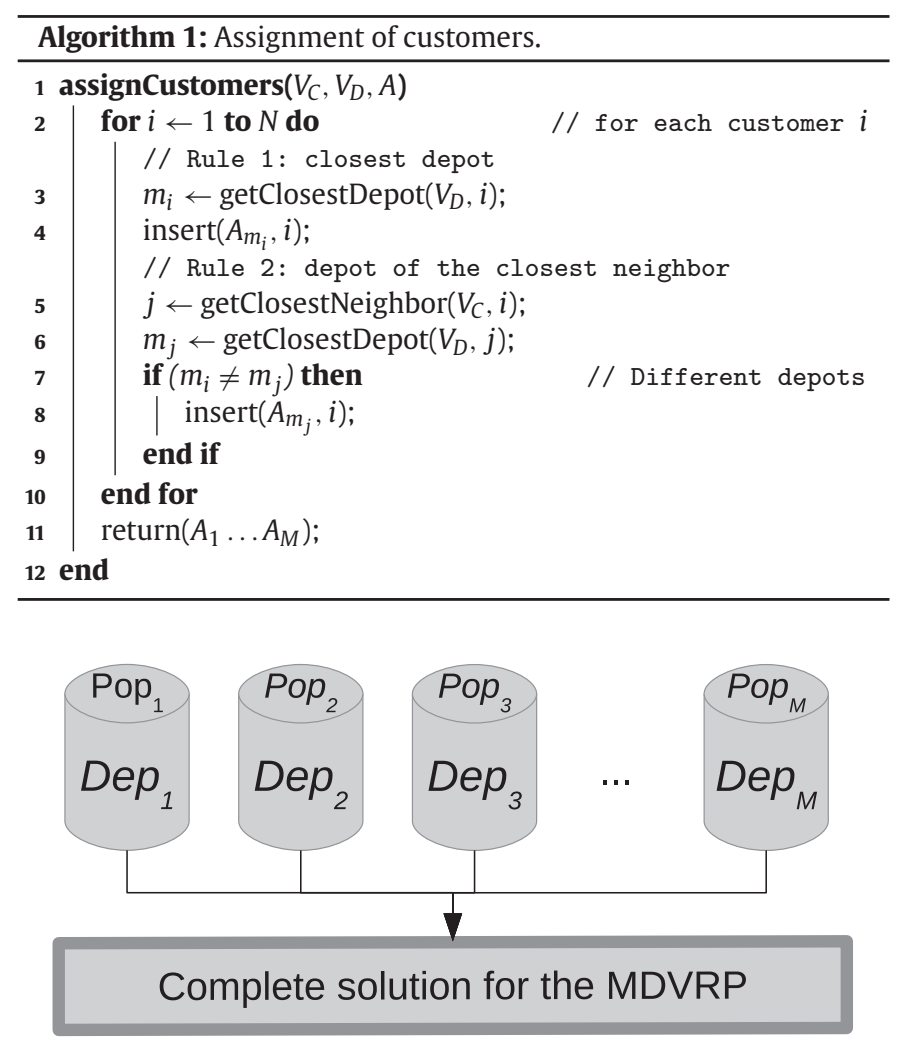

Fig. 4. Cooperative coevolutionary model for the MDVRP.

as well as the set of $\operatorname{arcs} A$. In Line 3 the closest depot $m_{i}$ to customer $i$ is selected according to rule 1 and inserted in the assignment group $A_{m_{i}}$ of depot $m_{i}$ (Line 4). The closest neighbor to customer $i$ is defined as customer $j$ (Line 5 ) and the closest depot to $j$, identified as $m_{j}$, is selected according to rule 2 . The depots are compared in Line 7. If the two depots are different $\left(m_{i} \neq m_{j}\right)$, customer $i$ is also inserted in the allocation group $A_{m_{j}}$ of depot $m_{j}$ (Line 8). After processing all customers, the assignment groups are returned (Line 11).

After this decomposition, each subproblem becomes a classical single depot VRP for a subset of customers identified by the two assignment rules above. Given that the gray nodes are duplicated, a repair operator will be needed to obtain a valid complete solution (see Section 5.5). Each subproblem is solved with an evolutionary algorithm, in which each individual represents a partial solution to the MDVRP. Fig. 4 illustrates the structure of the proposed coevolutionary model. For each depot, there is one population which evolves and searches the best routes for the set of customers assigned to it. Then, one individual for each population is selected to create a complete solution for the MDVRP.

A decomposition approach is particularly interesting for problem instances with a low degree of interdependency (coupling) between the subproblems. For example, customer 19 in Fig. 3 should clearly be served by depot C. It is unlikely that good solutions will be obtained by assigning this customer to depots $\mathrm{A}$ or $\mathrm{B}$, and these solutions are automatically eliminated through the decomposition approach. It is clear that some degree of interdependency exists among the subproblems for the instance illustrated in Fig. 3, due to the presence of gray nodes.

This model is strongly suitable for a parallel environment where each population evolves separately and cooperates with other populations to solve the problem. A parallel architecture for this model is proposed in the next section.
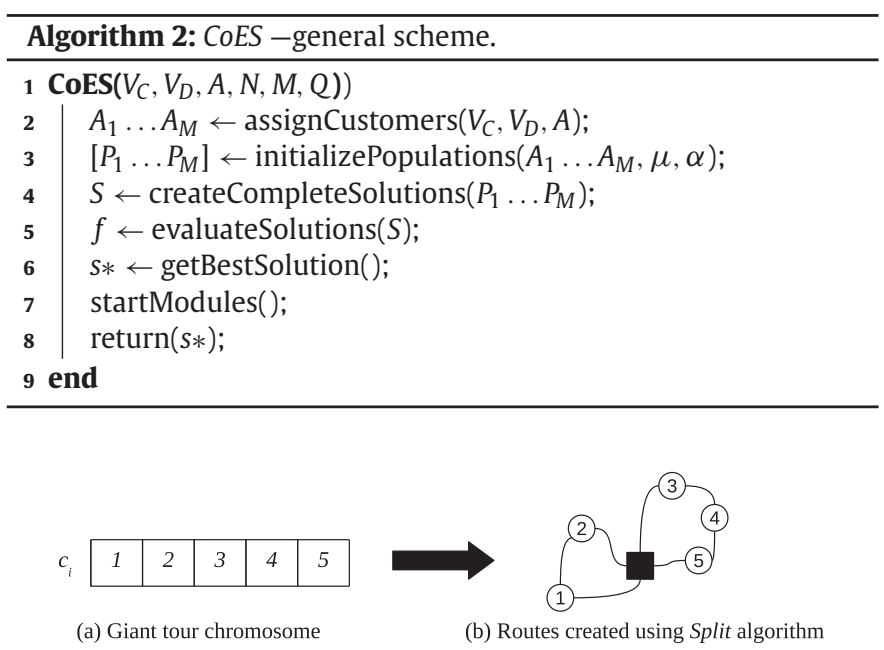

Fig. 5. Giant tour representation and the obtained routes.

\section{Parallel evolution strategy}

A parallel environment exploiting the evolution strategy (ES) paradigm, called CoES, supports the evolution of our cooperative coevolutionary model. Evolution strategy (Engelbrecht, 2007; Freitas, Guimarães, Pedrosa Silva, \& Souza, 2014; Luke, 2013) is an evolutionary algorithm using mutation as the main operator to generate new solutions. ES was chosen because each subproblem has a variable length representation (genotype) and the design of a recombination operator in this case would be rather cumbersome (see Section 5.2).

The proposed parallelization scheme, which is operational under asynchronous updates, is shown in Algorithm 2. CoES first receives all required information about the problem, in particular the number of customers $(N)$, number of depots $(M)$ and vehicle capacity $(Q)$. Each population is initialized with $\mu$ individuals (Line 3 ), which are encoded using the representation scheme presented in Section 5.1. The initialization procedure uses a semi-greedy method to insert customers from a given list. Parameter $\alpha$ defines the number of customers in this list, as discussed in Section 5.2. Complete solutions are then created with a Random fitness sampling strategy (Engelbrecht, 2007) (Line 4). Here, individuals from each initial population are selected randomly to create complete solutions. It should be noted that another strategy is used in the following populations, as explained in Section 5.5. In Line 5, all complete solutions are evaluated and the best one is selected (Line 6). Then, a number of parallel modules are started (Line 7). At the end, the best complete solution to the MDVRP is returned (Line 8).

The representation and initialization procedures are described in Sections 5.1 and 5.2. The parallel modules are introduced in Section 5.3.

\subsection{Representation}

Individuals from each population are represented by a giant tour, without route delimiters. It is basically a single sequence made of all customers assigned to a depot, as shown in Fig. 5(a). Since each individual in a population corresponds to a particular depot and subset of customers, the length of the giant tour is likely to change (variable genotype). Individual routes are created from this giant tour with the Split algorithm (Prins, 2004), which can optimally extract feasible routes from a single sequence. In constrained problems, the Split algorithm can be relaxed at the beginning to allow infeasible routes that violate one or more constraints. During the execution of the coevolutionary algorithm, this relaxation is progressively reduced to 

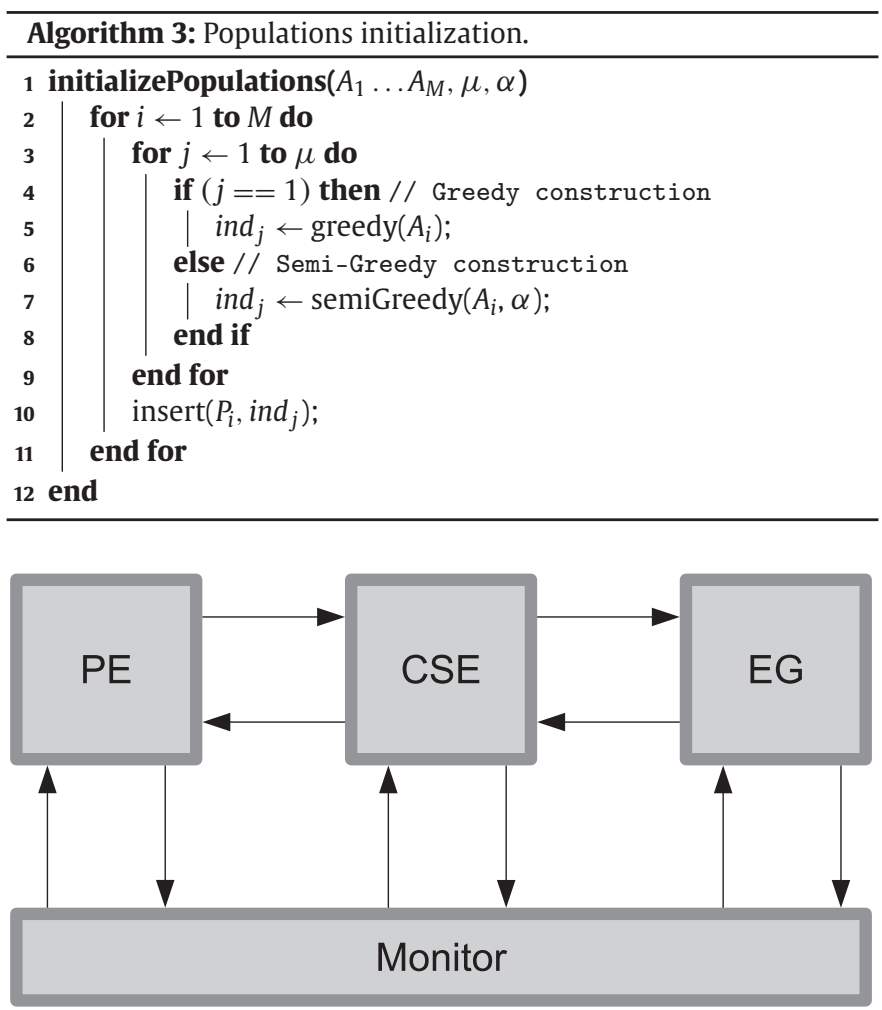

Fig. 6. Architecture of the parallel modules in CoES.

converge toward feasible routes. Fig. 5(b) illustrates two routes that could be obtained from the giant tour representation in 5(a).

\subsection{Initialization}

The population initialization procedure is shown in Algorithm 3 . The first individual in each population is constructed with the Nearest Insertion Heuristic (NIH) (Bodin, Golden, Assad, \& Ball, 1983) while the other ones are constructed with a semi-greedy approach based on NIH. With regard to the first individual, the closest customer to the depot is first inserted in the giant tour. Then, the next customer to be inserted is the one which is closest to the previous one. This is repeated until the giant tour is complete.

Based on this greedy heuristic, a semi-greedy variant generates the remaining individuals. The first customer is selected at random. Then, the remaining customers are sorted based on their distance from the previous one. A restricted candidate list (RCL) is created with the $\alpha$ best-ranked customers and the next customer to be inserted is selected at random in the RCL. This is repeated until the giant tour is complete.

\subsection{Parallel modules and coordination}

The parallel modules are executed until a stopping criterion, based on the execution time, is met. Fig. 6 depicts the architecture of these modules within CoES as well as their communication scheme.

The Start Modules procedure is shown in Algorithm 4 . It is called in Line 7 of Algorithm 2 to create a thread for each module and to initialize the environment. A start flag is used to indicate that each module should wait until all modules have been initialized. At the beginning of the procedure, the flag is set to FALSE (Line 2). At the end, the start flag is set to TRUE (Line 7) so that all modules can be executed.

The Monitor module manages the parallel processes and transmits information about the MDVRP problem. When the time-based stop-
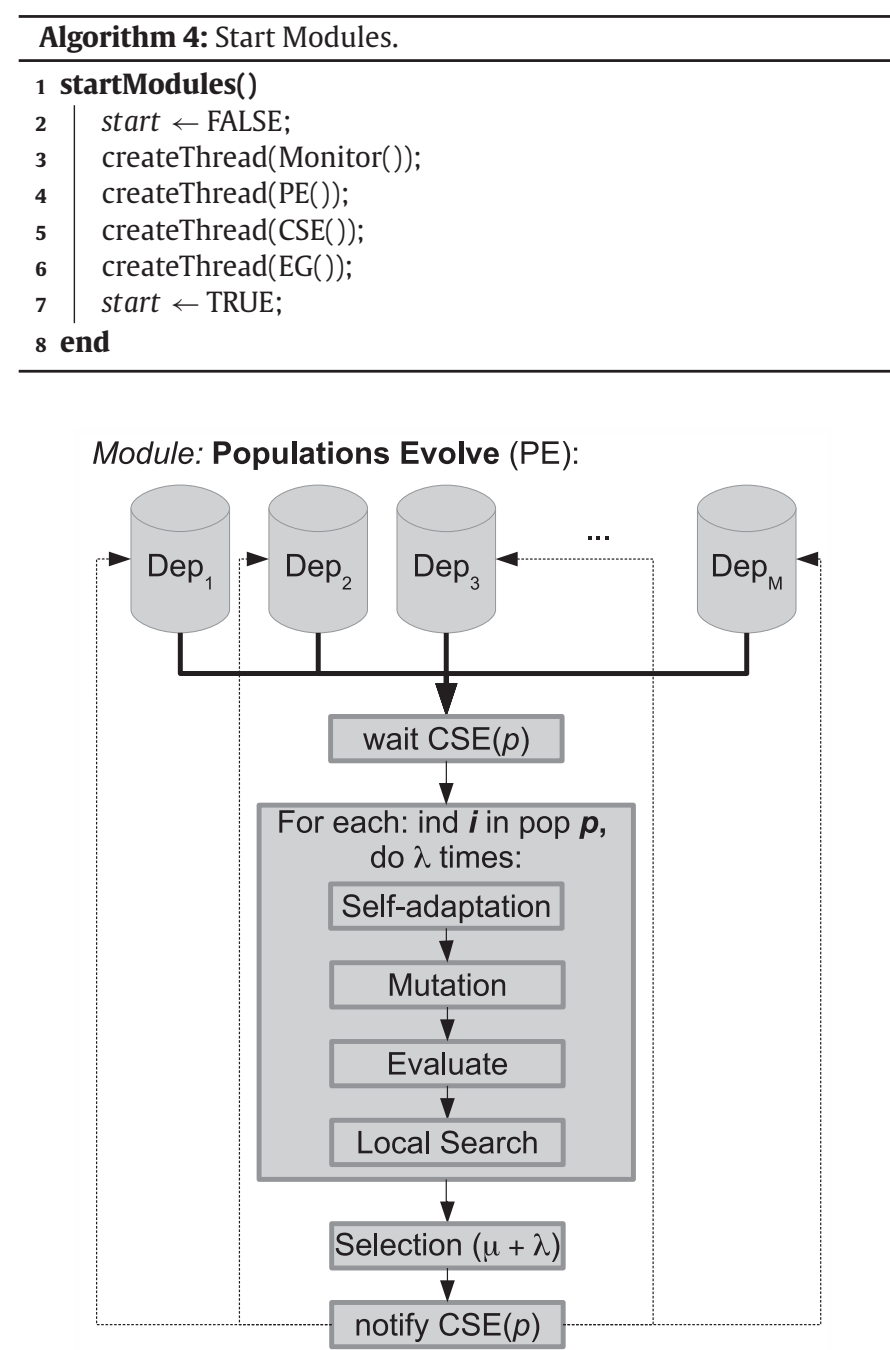

Fig. 7. Population Evolve module.

ping criterion is met, all modules are terminated by the Monitor module and the best solution is returned.

The Population Evolve (PE) module evolves each population with ES. The Complete Solutions Evaluate (CSE) module combines individuals from different populations to create and evaluate complete solutions. In addition, it applies local search heuristics to improve the complete solutions. The Elite Group (EG) module maintains an elite set of complete solutions, and also applies local search heuristics to these elite solutions. The various modules mentioned above are explained in detail in the following sections.

\subsection{Population Evolve (PE) module}

The Population Evolve (PE) module manages the ES-based evolution by creating a thread for each population. Within a thread, the evolution process is run sequentially. This is represented in Fig. 7. Note that the ES-based evolution is highlighted in the gray box of Fig. 7.

With regard to the ES-based population evolution, each one of the $\lambda$ offspring is generated as follows. First, a parent is selected at random, so that each parent generates $\lambda / \mu$ offspring on average. The self-adaptive procedure updates the number of mutations (strategy parameter $\sigma$ ) using a binomial distribution $B(n, p)$. The distribution is computed with $n$ equal to the number of customers in the giant tour (genotype length) and $p$ equal to 0.5 . The mutation is applied to the giant tour by selecting two different random positions and 


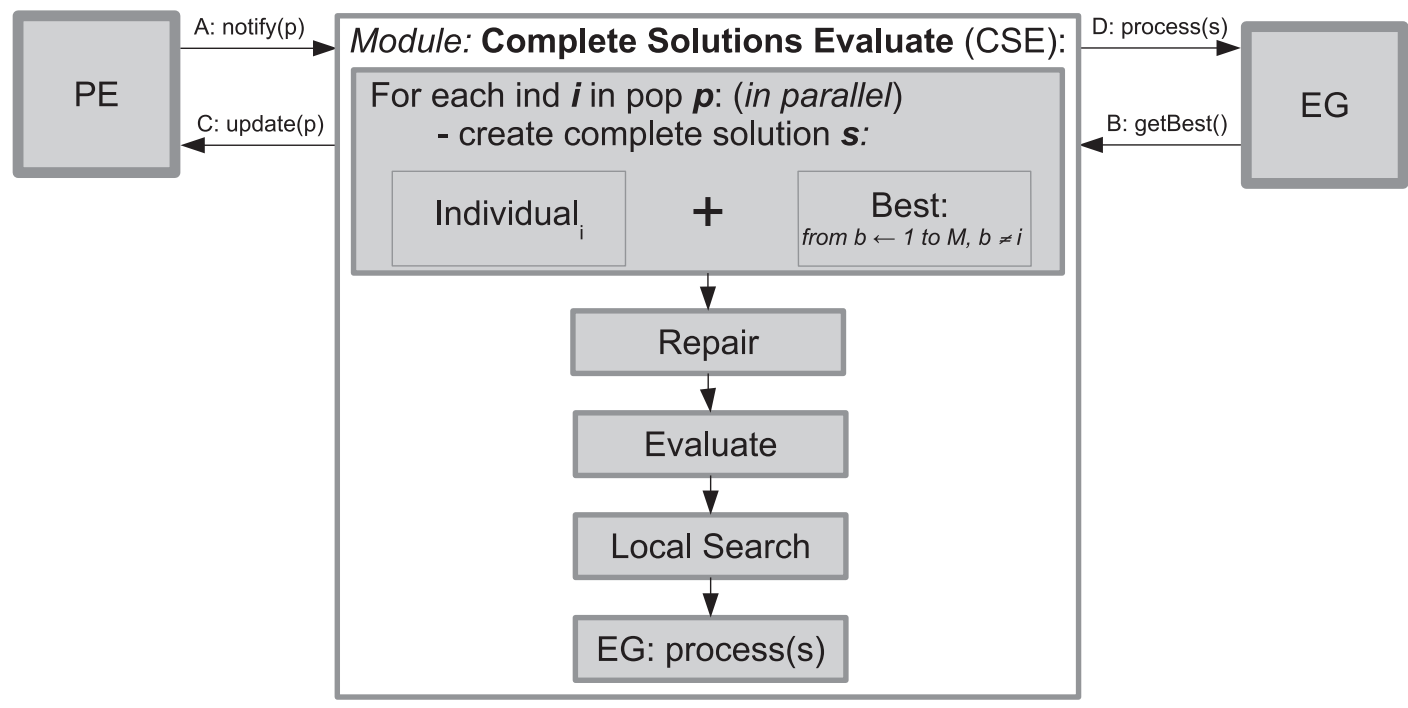

Fig. 8. Complete Solutions Evaluate module.

by swapping the corresponding customers. This is repeated $\sigma$ times. Then, the Split algorithm is applied and individual routes are created, thus allowing for a local, population-based, fitness evaluation of the newly generated partial solution. With regard to fitness evaluation, the fixed number of vehicles at the depot is accounted for in different ways depending on the status of the parent. Basically, if this number is not exceeded in the parent, then its offspring incur a penalty cost for each vehicle in excess (if any). If this number is already exceeded in the parent, then no penalty is incurred in its offspring. With this approach, a mix of solutions with and without extra vehicles is maintained throughout the search.

A local search procedure is applied to the offspring with probability $\rho_{\mathrm{ls}}$. Nine neighborhood structures are defined to improve the routes. They are the same as those presented in Prins (2004), Vidal et al. (2012), and Siqueira Ruela et al. (2013). It is important to note that the local search is performed at the population or subproblem level, therefore only routes starting and ending at the depot and visiting the subset of customers in the subproblem are considered. Let us suppose that $u$ and $v$ are two customer nodes belonging to the same or different routes, $x$ is the successor of $u$ and $y$ is the successor of $v$ along their respective routes. The following moves are applied in random order, and the exploration of the corresponding neighborhood stops as soon as an improving move is found:

- Move 1: move $u$ after $v$;

- Move 2: move $(u, x)$ after $v$;

- Move 3: move $(x, u)$ after $v$;

- Move 4: exchange $u$ and $v$;

- Move 5: exchange $(u, x)$ with $v$;

- Move 6: exchange $(u, x)$ and $(v, y)$;

- Move 7: if $(u, x)$ and $(v, y)$ are in the same route (but not adjacent), replace them by $(u, v)$ and $(x, y)$;

- Move 8: if $(u, x)$ and $(v, y)$ are in distinct routes, replace them by $(u, v)$ and $(x, y)$;

- Move 9: if $(u, x)$ and $(v, y)$ are in distinct routes, replace them by $(u, y)$ and $(v, x)$.

Move 7 is a 2-opt move, while moves 8 and 9 are 2-opt* moves. When all neighborhoods have been explored and no improvement to the current solution has been found, the local search procedure stops (Siqueira Ruela et al., 2013).

Once all offspring are created, parents and offspring are ranked according to their local fitness and the $\operatorname{ES}(\mu+\lambda)$ selection is applied to produce the next population. Using a producer-consumer synchro-

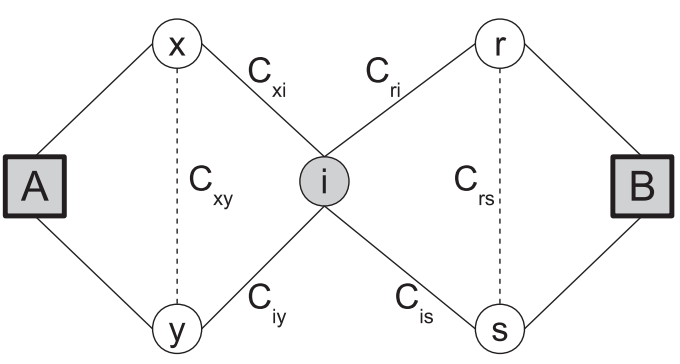

Fig. 9. Repair procedure-remove duplicates.

nization scheme, the new population is then processed by the CSE module. The corresponding thread waits until the end of the evaluation of all complete solutions before restarting.

\subsection{Complete Solutions Evaluate (CSE) module}

The Complete Solutions Evaluate module receives a population of partial solutions from the PE module and evaluates the global fitness of complete solutions. Each complete solution is created and evaluated in a multithread environment, where a thread is started for each individual $i$ in population $p$, as illustrated in Fig. 8.

The CSE module is notified by the PE module to process population $p$ at instant $\mathrm{A}$ in Fig. 8. It combines each individual in $p$ with the best individuals obtained from the other populations to create a complete solution. More precisely, the partial solutions associated with the other depots are taken from the best solution in the Elite Group module (All versus best strategy), see instant B in Fig. 8.

Once a complete solution is created, a repair procedure is applied to remove duplicate customers and to insert customers that are not included in the solution. In both cases, the least additional cost heuristic is used (Bodin et al., 1983). The additional cost when customer $i$ is between customers $x$ and $y$ is defined in Eq. (12). The least additional cost is looked for when inserting a customer between two consecutive customers in the solution. When duplicates must be removed, only the copy with least additional cost is kept.

$C_{x i y}=c_{x i}+c_{i y}-c_{x y}$

To illustrate the removal of a duplicate customer, we refer to Fig. 9 where customer $i$ is included in two routes associated with depots $A$ and $B$. The additional cost is calculated in both cases with Eq. (12) to obtain $C_{x i y}$ for depot A and $C_{r i s}$ for depot B. If $C_{x i y}<C_{r i s}$, then customer 


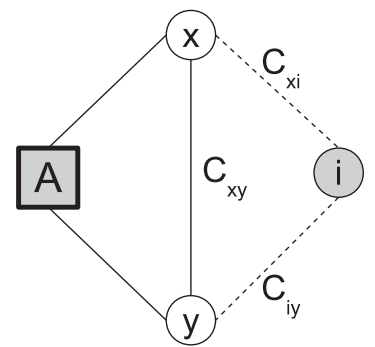

Fig. 10. Repair procedure-insert customer.

Module: Elite Group (EG):

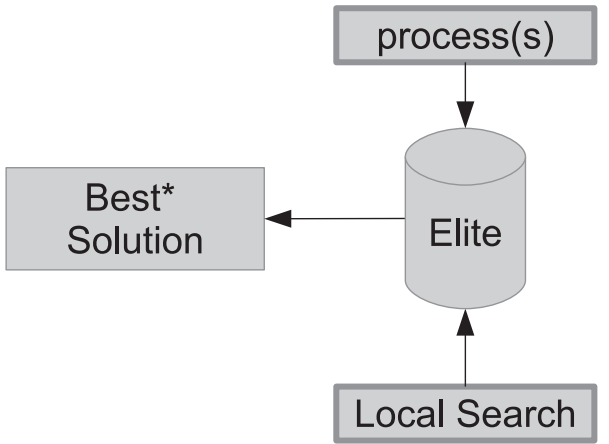

Fig. 11. Elite Group module.

$i$ is kept in the route of depot $A$ and the duplicate is removed from the route of depot $B$.

When inserting a customer in a solution, all insertion positions between two consecutive customers are considered and the least cost one is chosen. In Fig. 10, customer $i$ is inserted between customers $x$ and $y$ in a route of $\operatorname{depot} A$.

The complete solution is evaluated and submitted to a local search procedure with probability $\rho_{\mathrm{ls}}$. The local search is based on the same moves than those presented in Section 5.4. However, it is now possible for a customer to be moved from one depot to another, thus changing individuals in the populations. This is the reason for the population update at instant $C$ in Fig. 8. After the update, the PE module is notified and can restart.

Each complete solution created is sent to the EG module at instant D in Fig. 8. The module checks if the complete solution can be part of the elite group, as it is explained next.

\subsection{Elite Group (EG) module}

The Elite Group (EG) module maintains a set of $\tau$ elite complete solutions, including the best solution to the MDVRP found so far. This is illustrated in Fig. 11. Assuming that a complete solution is submitted by the CSE module, the EG module will try to add the solution to the elite group. If there are less than $\tau$ solutions in the elite group, the solution is automatically added. Otherwise, the new solution can enter the elite group only if it is better than the worst solution in the group, in which case it replaces the latter.

The local search for the elite group operates in two steps, called PR and RI, where PR stands for Path Relinking and RI for Route Improvement. The local search is run in a multithread environment with a thread for each solution in the elite group.

PR uses each solution in the elite group as an initial solution and the best solution so far as the guiding solution. To apply PR in this setting, the difference between an initial solution $i$ and the guiding solution $g$ must be calculated. We assume that each customer in a solution is characterized by the vehicle serving this customer and the corresponding depot. For each customer, the triplet (customer, depot,

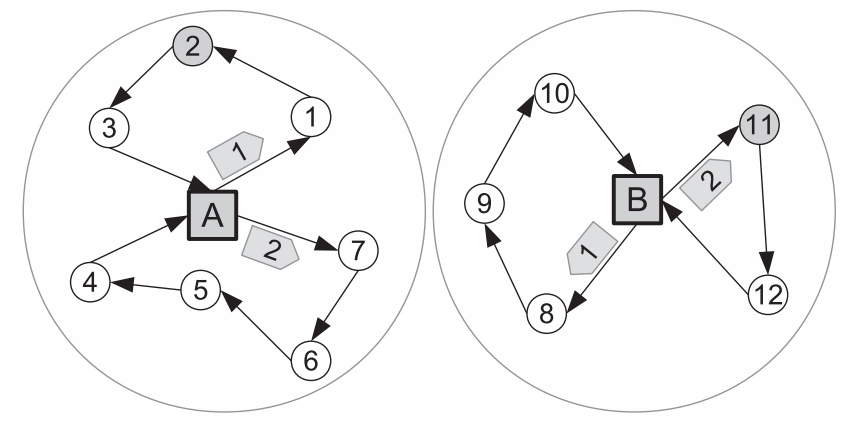

(a) Initial solution

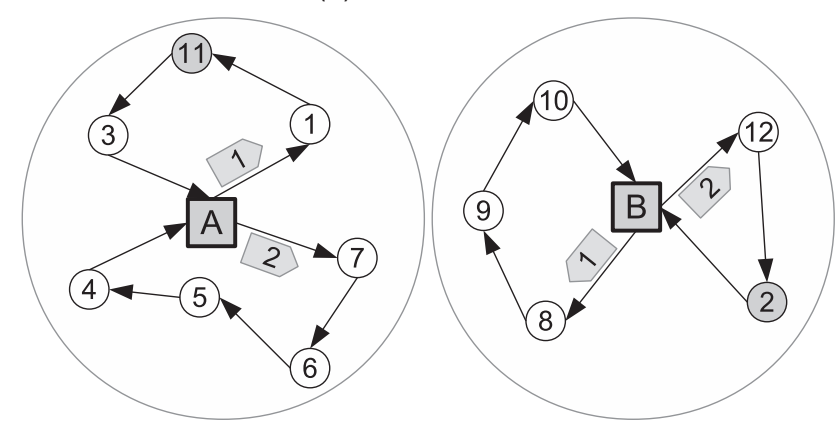

(b) Guiding solution

Fig. 12. Path-Relinking!.

vehicle) from the guiding solution is added to set $\Delta$ when it differs from the initial solution.

We refer to Fig. 12 for an example. In this figure, we have one initial solution (Fig. 12a) and a guiding solution (Fig. 12b). There are two depots A and B, 12 customers and two vehicles at each depot (represented by the pentagons numbered 1 and 2). The difference between the initial and guiding solutions corresponds to the nodes in gray, namely customers 2 and 11 . Thus $\Delta=\{(2, B, 2),(11, A, 1)\}$. The triplets in $\Delta$ are then processed one by one to progressively modify the initial solution. That is, the customer in the first triplet is removed from its route in the initial solution and reinserted in the route indicated by the depot and vehicle of the guiding solution. A new solution is obtained which is closer to the guiding solution. The procedure is then applied again using the new solution and the second triplet. This is repeated until all triplets are done. It is hoped that a solution better than the guiding solution will emerge along the path between the initial and guiding solutions.

The RI improvement procedure is applied to the solution returned by PR. This procedure is the local search mentioned in Section 5.5. If the solution obtained at the end is better than the best solution, then the elite group is updated.

\section{Computational results}

The performance of the proposed CoES algorithm was assessed through a number of computational experiments. They were conducted on the 33 MDVRP benchmark instances taken from the literature (Cordeau et al., 1997; Cordeau \& Maischberger, 2012; Escobar et al., 2014; Subramanian et al., 2013; Vidal et al., 2012). ${ }^{1}$ These instances are Euclidean and the time units are the same as the distance

\footnotetext{
1 The MDVRP instances might also be obtained in https://github.com/ fboliveira/MDVRP-Instances
} 
Table 1

Selected parameter values.

\begin{tabular}{lll}
\hline Parameter & Lower bound & Upper bound \\
\hline$T_{\max }$ & $600 \mathrm{~s}$ & $1800 \mathrm{~s}$ \\
$T_{\text {upd }}$ & $300 \mathrm{~s}$ & $600 \mathrm{~s}$ \\
$\mu$ & 20 & 100 \\
$\tau$ & 5 & 50 \\
$\rho_{\mathrm{ls}}$ & 0.2 & 0.8 \\
\hline
\end{tabular}

units. They are divided into S1 $(P 01-P 23)$ and S2 $(P 24-P 33)^{2}$ and have various sizes ranging from 48 to 360 customers, see Table 4 .

The algorithm was implemented in $\mathrm{C}++11^{3}$ and all experiments were run on a computer with two $2.50 \mathrm{GHz}$ Intel Xeon (E5-2640) processors with 12 cores per CPU and 96 GB RAM. The computer was run under the Ubuntu 14.04.1 LTS operational system.

A first experiment was realized to set the parameter values, as it is discussed in Section 6.1. A comparison with the best solutions reported in the literature follows in Section 6.2.

\subsection{Parameter settings}

The algorithm stops when it reaches either the allowed total computation time $T_{\max }$ or the maximum computation time without improvement to the best solution $T_{\text {upd }}$. Also, the number of individuals $\mu$ in each population and the maximum number of complete solutions in the elite group $\tau$ must be defined. Finally, the local search procedure in the PE and CSE modules are applied with a probability $\rho_{\mathrm{ls}}$.

After some preliminary tests and using some values commonly found in the literature, lower and upper bound values were determined for the above parameters. These values are presented in Table 1.

As there are two values (levels) for each parameter (factors), a $2^{k}$ factorial experiment was designed to study the impact of each factor as well as their interactions. There are five factors with two levels each, resulting in 32 observations $\left(2^{5}\right)$ for each instance. Selecting the instance in group S1 (P01 - P23) from the benchmark, we obtain a total of 736 observations (i.e., $32 \times 23$ ). Given the large number of factors and total observations, an experiment with a single replication was performed. In this situation, the Sparsity-of-effects principle applies, that is, the main effects and low-order interactions usually dominate. Then, we can use an experiment with one replication and combine highest-order interaction for calculating the mean square error (Campelo, 2015, Chap. 12; Montgomery \& Runger, 2011), The response variable corresponds to the gap between the solution value obtained by CoES and the best-known solution value reported in Vidal et al. (2012).

The experiment is performed with the analysis of variance (ANOVA) method from the R-Project (Core Team, 2015). Among other assumptions, ANOVA requires a normal distribution for the residuals. As the number of samples is large, they are approximately normal because of the Central Limit Theorem (CLT) and this assumption is satisfied.

Table 2 reports only the significant factors and interactions identified by ANOVA. In this case, the null hypothesis states that there is no significant impact and a $p$-value smaller than 0.05 invalidates this assumption. Table 2 shows the significant factors identified by ANOVA along with their corresponding $p$-value.

Two main factors and one interaction have a significant impact on the results. The most significant factor is the number of individuals in each population $\mu$. The second one is the elite group size $\tau$. The third

\footnotetext{
2 Those instances were proposed by Cordeau et al. (1997) and they are also named pr01-pr10.

${ }^{3}$ C++ 2011 Standard.
}

Table 2

Significant factors and interactions.

\begin{tabular}{ll}
\hline Factor/interaction & $p$-value \\
\hline$\mu$ & $<2^{-16}$ \\
$\tau$ & 0.035 \\
$\mu: \rho_{\mathrm{ls}}: \tau$ & 0.016 \\
\hline
\end{tabular}

Table 3

Final parameter values.

\begin{tabular}{ll}
\hline Parameter & Value \\
\hline$T_{\max }$ & $1800 \mathrm{~s}$ \\
$T_{\text {upd }}$ & $600 \mathrm{~s}$ \\
$\mu$ & 20 \\
$\tau$ & 50 \\
$\rho_{\text {ls }}$ & 0.2 \\
\hline
\end{tabular}
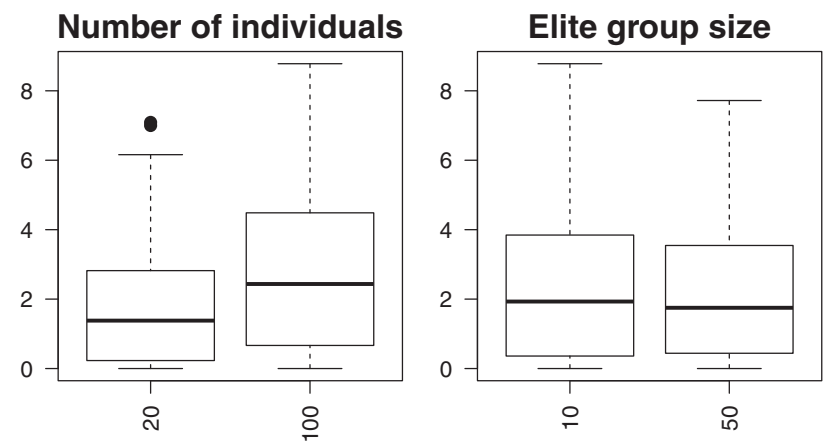

Fig. 13. Boxplots of the two main factors.
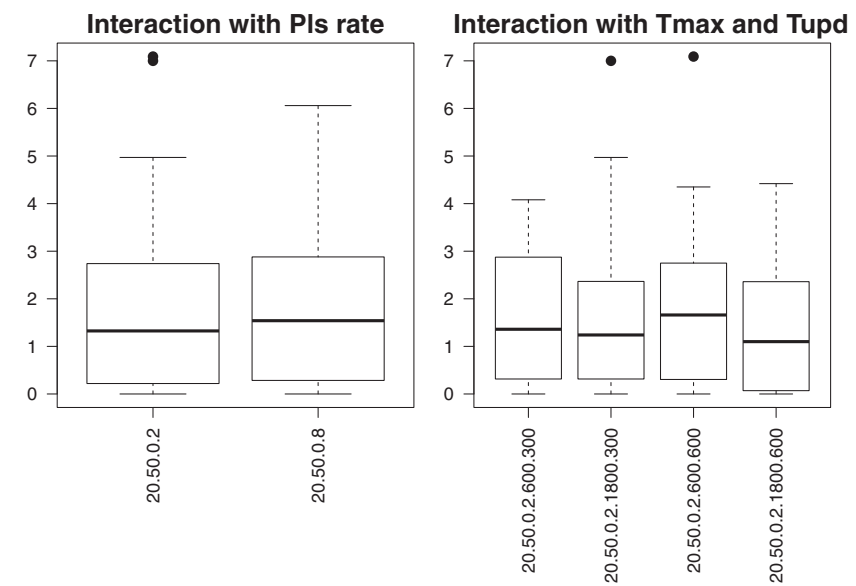

Fig. 14. Boxplots of interactions.

one corresponds to the interaction of the two previous factors with the local search rate $\rho_{\mathrm{ls}}$. Fig. 13 shows box plots of the main factors. Based on these plots, $\mu$ was set to 20 and $\tau$ was set to 50 .

Then, the other parameters can be set by fixing $\mu$ and $\tau$ to the above values and by observing the interactions. Fig. 14 shows boxplots of the interaction of $\mu=20$ and $\tau=50$ with $\rho_{\mathrm{ls}}, T_{\max }$ and $T_{\text {upd }}$, respectively. The parameter values associated with the first two boxplots are shown in the format $\mu . \tau$. $\rho_{\mathrm{ls}}$. In the case of the four last boxplots, the format is $\mu . \tau$. $\rho_{\mathrm{ls}} \cdot T_{\max } \cdot T_{\mathrm{upd}}$. The interaction between $\mu, \tau$ and $\rho_{\mathrm{ls}}$ was shown to be a significant factor in Table 2. Fig. 14 indicates that an appropriate value for $\rho_{\mathrm{ls}}$ is 0.2 . With regard to $T_{\max }$ and $T_{\mathrm{upd}}$, Fig. 14 shows that suitable values are $T_{\max }=1800$ and $T_{\text {upd }}=600$. 
Table 4

$\operatorname{CoES}(\lambda=\mu)$ : results.

\begin{tabular}{|c|c|c|c|c|c|c|c|}
\hline \multirow[t]{2}{*}{ Inst } & \multirow[t]{2}{*}{$M$} & \multirow[t]{2}{*}{$N$} & \multirow[t]{2}{*}{$Q$} & \multirow[t]{2}{*}{$T$} & \multicolumn{2}{|c|}{ Average values } & \multirow[t]{2}{*}{ Best cost } \\
\hline & & & & & Time (s) & Cost & \\
\hline P01 & 4 & 50 & 80 & $\infty$ & 1.00 & $\left(^{*}\right) 576.87$ & 576.87 \\
\hline P02 & 2 & 50 & 160 & $\infty$ & 0.50 & 475.06 & 473.87 \\
\hline P03 & 3 & 75 & 140 & $\infty$ & 2.50 & 643.57 & 641.19 \\
\hline P04 & 8 & 100 & 100 & $\infty$ & 189.70 & 1011.42 & 1007.40 \\
\hline P05 & 5 & 100 & 200 & $\infty$ & 26.60 & 752.39 & 750.11 \\
\hline P06 & 6 & 100 & 100 & $\infty$ & 77.30 & 877.86 & 876.50 \\
\hline P07 & 4 & 100 & 100 & $\infty$ & 24.20 & 893.36 & 888.41 \\
\hline P08 & 14 & 249 & 500 & 310 & 803.40 & 4474.23 & 4450.37 \\
\hline P09 & 12 & 249 & 500 & 310 & 513.30 & 3904.92 & 3895.70 \\
\hline P10 & 8 & 249 & 500 & 310 & 719.90 & 3680.02 & 3666.35 \\
\hline P11 & 6 & 249 & 500 & 310 & 396.20 & 3593.37 & 3569.68 \\
\hline P12 & 5 & 80 & 60 & $\infty$ & 0.90 & $\left({ }^{*}\right) 1318.95$ & 1318.95 \\
\hline P13 & 5 & 80 & 60 & 200 & 0.00 & $\left(^{*}\right) 1318.95$ & 1318.95 \\
\hline P14 & 5 & 80 & 60 & 180 & 0.00 & $\left({ }^{*}\right) 1360.12$ & 1360.12 \\
\hline P15 & 5 & 160 & 60 & $\infty$ & 107.00 & 2549.65 & 2526.06 \\
\hline P16 & 5 & 160 & 60 & 200 & 8.20 & $\left({ }^{*}\right) 2572.23$ & 2572.23 \\
\hline P17 & 5 & 160 & 60 & 180 & 14.70 & 2733.80 & 2709.09 \\
\hline P18 & 5 & 240 & 60 & $\infty$ & 429.10 & 3781.66 & 3771.35 \\
\hline P19 & 5 & 240 & 60 & 200 & 72.60 & $\left(^{*}\right) 3827.06$ & 3827.06 \\
\hline P20 & 5 & 240 & 60 & 180 & 190.20 & 4094.86 & 4058.07 \\
\hline P21 & 5 & 360 & 60 & $\infty$ & 554.90 & 5668.97 & 5608.26 \\
\hline P22 & 5 & 360 & 60 & 200 & 214.00 & 5708.78 & 5702.16 \\
\hline P23 & 5 & 360 & 60 & 180 & 529.30 & 6159.90 & 6129.99 \\
\hline P24 & 1 & 48 & 200 & 500 & 0.00 & 849.17 & 849.17 \\
\hline P25 & 2 & 96 & 195 & 480 & 630.80 & 1271.39 & 1269.56 \\
\hline P26 & 3 & 144 & 190 & 460 & 123.60 & 1768.13 & 1759.77 \\
\hline P27 & 4 & 192 & 185 & 440 & 580.80 & 2057.50 & 2041.76 \\
\hline P28 & 5 & 240 & 180 & 420 & 321.00 & 2362.75 & 2314.72 \\
\hline P29 & 6 & 288 & 175 & 400 & 724.40 & 2690.01 & 2674.53 \\
\hline P30 & 1 & 72 & 200 & 500 & 0.60 & $\left({ }^{*}\right) 1070.85$ & 1070.85 \\
\hline P31 & 2 & 144 & 190 & 475 & 254.00 & 1650.96 & 1633.34 \\
\hline P32 & 3 & 216 & 180 & 450 & 652.50 & 2160.76 & 2139.78 \\
\hline \multirow[t]{4}{*}{ P33 } & 4 & 288 & 170 & 425 & 472.80 & 2844.32 & 2825.90 \\
\hline & \multicolumn{3}{|c|}{ Average } & & 261.70 & 2445.57 & 2432.67 \\
\hline & \multicolumn{3}{|c|}{$\mathrm{S} 1$} & & 211.98 & 2694.69 & 2682.55 \\
\hline & \multicolumn{3}{|c|}{ S2 } & & 376.05 & 1872.58 & 1857.94 \\
\hline
\end{tabular}

The final parameter settings for the CoES algorithm are summarized in Table 3. The comparison with other algorithms in the next section is based on these parameter values.

\subsection{Comparison with other methods}

The performance of our CoES was compared with the best heuristics proposed for the MDVRP, namely, the Tabu Search (CGL) (Cordeau et al., 1997), the adaptive large neighborhood search (ALNS) (Pisinger \& Ropke, 2007), a fuzzy logic guided genetic algorithm (FLGA) (Lau, Chan, Tsui, \& Pang, 2010), a parallel iterated Tabu Search heuristic (ITS) (Cordeau \& Maischberger, 2012), a hybrid algorithm combining Iterated Local Search and Set Partitioning (ILS-RVND-SP) (Subramanian et al., 2013), a hybrid genetic algorithm with adaptive diversity control (HGSADC+) (Vidal et al., 2014) and a hybrid Granular Tabu Search (ELTG) (Escobar et al., 2014).

CoES was run 10 times on all benchmark instances, using the previously defined parameter settings. To remove any factor that could impact the performance, the order of execution of all replicates was randomized. Note that heuristics CGL and ELTG were run only once on each instance and the value reported was used to compare with the best value of CoES.

The results from CoES are shown in Table 4. The instance name is in column Inst, $M$ is the number of depots, $N$ is the number of customers, $Q$ is the vehicle capacity and $T$ is the maximum duration time of a vehicle route ( $\infty$ means that there is no time constraint). The next two columns present the average values for the processing time (in seconds) and for the solution cost. When $(*)$ appears, the same
Table 5

$\operatorname{CoES}(\lambda=\mu)$ : comparison with results of the literature-best values.

\begin{tabular}{|c|c|c|c|c|c|}
\hline Instance & CGL & ITS & ILS-RVND-SP & HGSADC+ & ELTG \\
\hline P01 & 0.00 & 0.00 & 0.00 & 0.00 & 0.00 \\
\hline P02 & 0.00 & 0.07 & 0.07 & 0.07 & 0.07 \\
\hline P03 & -0.61 & 0.00 & 0.00 & 0.08 & 0.00 \\
\hline P04 & 0.07 & 0.64 & 0.64 & 0.82 & 0.64 \\
\hline P05 & -0.43 & 0.01 & 0.01 & 0.01 & 0.01 \\
\hline P06 & -0.15 & 0.00 & 0.00 & 0.00 & 0.00 \\
\hline P07 & -0.40 & 0.73 & 0.73 & 0.73 & 0.42 \\
\hline P08 & -0.72 & 1.41 & 1.62 & 1.77 & 1.80 \\
\hline P09 & -0.64 & 0.90 & 0.94 & 0.96 & 0.38 \\
\hline P10 & -1.30 & 0.97 & 0.96 & 0.97 & 1.01 \\
\hline P11 & -0.31 & 0.62 & 0.67 & 0.67 & 0.69 \\
\hline P12 & 0.00 & 0.00 & 0.00 & 0.00 & 0.00 \\
\hline P13 & 0.00 & 0.00 & 0.00 & 0.00 & 0.00 \\
\hline P14 & 0.00 & 0.00 & 0.00 & 0.00 & 0.00 \\
\hline P15 & -0.32 & 0.82 & 0.82 & 0.82 & 0.82 \\
\hline P16 & 0.00 & 0.00 & 0.00 & 0.00 & 0.00 \\
\hline P17 & -0.41 & 0.00 & 0.00 & 0.00 & 0.00 \\
\hline P18 & 1.64 & 1.85 & 1.85 & 1.85 & 1.85 \\
\hline P19 & 0.00 & 0.00 & 0.00 & 0.00 & 0.00 \\
\hline P20 & 0.00 & 0.00 & 0.00 & 0.00 & 0.00 \\
\hline P21 & 1.31 & 2.44 & 2.44 & 2.44 & 2.44 \\
\hline P22 & -0.24 & 0.00 & 0.00 & 0.00 & 0.00 \\
\hline P23 & -0.16 & 0.84 & 0.84 & 0.84 & 0.57 \\
\hline P24 & -1.41 & -1.41 & -1.41 & -1.41 & -1.41 \\
\hline P25 & -3.45 & -2.89 & -2.89 & -2.89 & -3.17 \\
\hline P26 & -3.08 & -2.44 & -2.44 & -2.44 & -2.44 \\
\hline P27 & -2.51 & -0.80 & -0.80 & -0.85 & -1.08 \\
\hline P28 & -3.88 & -0.71 & -0.71 & -1.09 & -1.49 \\
\hline P29 & -3.38 & -0.12 & -0.23 & -0.28 & -1.32 \\
\hline P30 & -1.95 & -1.72 & -1.72 & -1.72 & -1.72 \\
\hline P31 & -2.56 & -1.89 & -1.89 & -1.90 & -1.93 \\
\hline P32 & -1.70 & 0.31 & 0.31 & 0.26 & -0.54 \\
\hline P33 & -8.54 & -1.82 & -1.68 & -2.10 & -2.92 \\
\hline Average & -1.06 & -0.07 & -0.06 & -0.07 & -0.22 \\
\hline $\mathrm{S} 1$ & -0.12 & 0.49 & 0.50 & 0.52 & 0.47 \\
\hline S2 & -3.25 & -1.35 & -1.35 & -1.44 & -1.80 \\
\hline
\end{tabular}

solution was produced by CoES in each of the 10 runs. The last column shows the best solution cost obtained by CoES.

The best and mean solution values, when compared with the other methods, are shown in Tables 5 and 6, respectively. These tables report the gap (in \%), that is,

$100 \times \frac{\left(Z_{\text {CoES }}-Z_{\text {lit }}\right)}{Z_{\text {lit }}}$

where $Z_{\text {CoES }}$ is the solution value of CoEs and $Z_{\text {lit }}$ the value of one of the other methods (as reported in the literature). A negative value indicates that CoES performs better. The Average line reports the average gap over all instances. The lines $S 1$ and $S 2$ show the average gap over the instances in each subset. In the tables, the entries in bold indicate that the same or better solution values were obtained by CoES over the corresponding method.

With regard to the solutions reported in Table 4 labeled with $(*)$, it should be noted that the gap with the other methods in Table 6 for those instances is always null (gap $=0.00$ ) or negative (gap $<0.00$ ). When considering the gap between CoES and the other methods in Table 6 for the mean values, the average over all test instances is always smaller than $0.5 \%$. It is less than $0.9 \%$ for subset $\mathrm{S} 1$, while it is always negative for S2, indicating that CoES provides an improvement over each method in this case. When considering the gap in Table 5 for the best values, the average over all test instances is always negative. It indicates that CoES provides an improvement over every method. The trend observed for the mean values is also observed here: there is generally a positive gap in the case of subset S1, but a more important negative gap for S2. Thus, we conclude that CoES performs better on subset $\mathrm{S} 2$.

As HGSADC+ uses an evolutionary approach, some analyses have been performed using average costs and time for that method as a 
Table 6

$\operatorname{CoES}(\lambda=\mu)$ : comparison with results of the literature-mean values.

\begin{tabular}{|c|c|c|c|c|c|c|c|}
\hline Instance & CGL & ALNS & FLGA & ITS & ILS-RVND-SP & HGSADC+ & ELTG \\
\hline P01 & 0.00 & 0.00 & 0.00 & 0.00 & 0.00 & 0.00 & 0.00 \\
\hline P02 & 0.25 & 0.32 & 0.32 & 0.32 & 0.32 & 0.32 & 0.32 \\
\hline P03 & -0.25 & 0.37 & 0.37 & 0.37 & 0.37 & 0.46 & 0.37 \\
\hline P04 & 0.47 & 0.53 & 0.98 & 1.01 & 1.04 & 1.08 & 1.04 \\
\hline P05 & -0.13 & 0.01 & 0.04 & 0.31 & 0.29 & 0.31 & 0.31 \\
\hline P06 & 0.00 & -0.58 & -0.55 & 0.16 & 0.16 & 0.16 & 0.16 \\
\hline P07 & 0.16 & 0.45 & 0.61 & 1.29 & 1.29 & 1.29 & 0.98 \\
\hline P08 & -0.18 & 1.20 & 0.81 & 1.60 & 1.83 & 2.07 & 2.35 \\
\hline P09 & -0.41 & 0.32 & -0.29 & 0.92 & 1.05 & 1.14 & 0.62 \\
\hline P10 & -0.93 & 0.36 & 0.28 & 1.17 & 1.25 & 1.33 & 1.39 \\
\hline P11 & 0.35 & 0.56 & 0.32 & 1.27 & 1.33 & 1.30 & 1.36 \\
\hline P12 & 0.00 & -0.01 & 0.00 & 0.00 & 0.00 & 0.00 & 0.00 \\
\hline P13 & 0.00 & 0.00 & 0.00 & 0.00 & 0.00 & 0.00 & 0.00 \\
\hline P14 & 0.00 & 0.00 & 0.00 & 0.00 & 0.00 & 0.00 & 0.00 \\
\hline P15 & 0.61 & 1.19 & 1.42 & 1.77 & 1.77 & 1.77 & 1.77 \\
\hline P16 & 0.00 & -0.07 & -0.24 & 0.00 & 0.00 & 0.00 & 0.00 \\
\hline P17 & 0.50 & 0.91 & 0.91 & 0.91 & 0.87 & 0.91 & 0.91 \\
\hline P18 & 1.92 & 1.21 & 1.43 & 2.13 & 2.13 & 2.13 & 2.13 \\
\hline P19 & 0.00 & -0.30 & -0.35 & 0.00 & -0.01 & 0.00 & 0.00 \\
\hline P20 & 0.91 & 0.74 & 0.78 & 0.91 & 0.91 & 0.91 & 0.91 \\
\hline P21 & 2.40 & 3.04 & 2.59 & 3.55 & 3.55 & 3.55 & 3.55 \\
\hline P22 & -0.13 & -0.23 & -0.42 & 0.12 & 0.05 & 0.12 & 0.12 \\
\hline P23 & 0.33 & 1.10 & 1.00 & 1.33 & 1.33 & 1.31 & 1.06 \\
\hline P24 & -1.41 & -1.41 & -1.41 & -1.41 & -1.41 & -1.41 & -1.41 \\
\hline P25 & -3.32 & -2.75 & -3.16 & -2.75 & -2.84 & -2.75 & -3.03 \\
\hline P26 & -2.62 & -2.10 & -2.30 & -2.00 & -1.99 & -1.98 & -1.98 \\
\hline P27 & -1.75 & -0.17 & -0.90 & -0.17 & -0.17 & -0.04 & -0.32 \\
\hline P28 & -1.88 & 1.07 & -1.20 & 1.09 & 1.05 & 1.35 & 0.56 \\
\hline P29 & -2.82 & 0.09 & -1.43 & 0.26 & 0.18 & 0.51 & -0.75 \\
\hline P30 & -1.95 & -1.72 & -1.72 & -1.72 & -1.72 & -1.72 & -1.72 \\
\hline P31 & -1.51 & -0.83 & -0.94 & -0.87 & -0.85 & -0.83 & -0.87 \\
\hline P32 & -0.74 & 1.14 & 0.40 & 1.02 & 1.19 & 1.29 & 0.43 \\
\hline P33 & -7.94 & -1.57 & -2.58 & -1.47 & -1.32 & -0.83 & -2.28 \\
\hline Average & -0.61 & 0.09 & -0.16 & 0.34 & 0.35 & 0.42 & 0.24 \\
\hline S1 & 0.26 & 0.48 & 0.44 & 0.83 & 0.85 & 0.88 & 0.84 \\
\hline S2 & -2.59 & -0.83 & -1.52 & -0.80 & -0.79 & -0.64 & -1.14 \\
\hline
\end{tabular}

\section{Average GAP x Ratio}

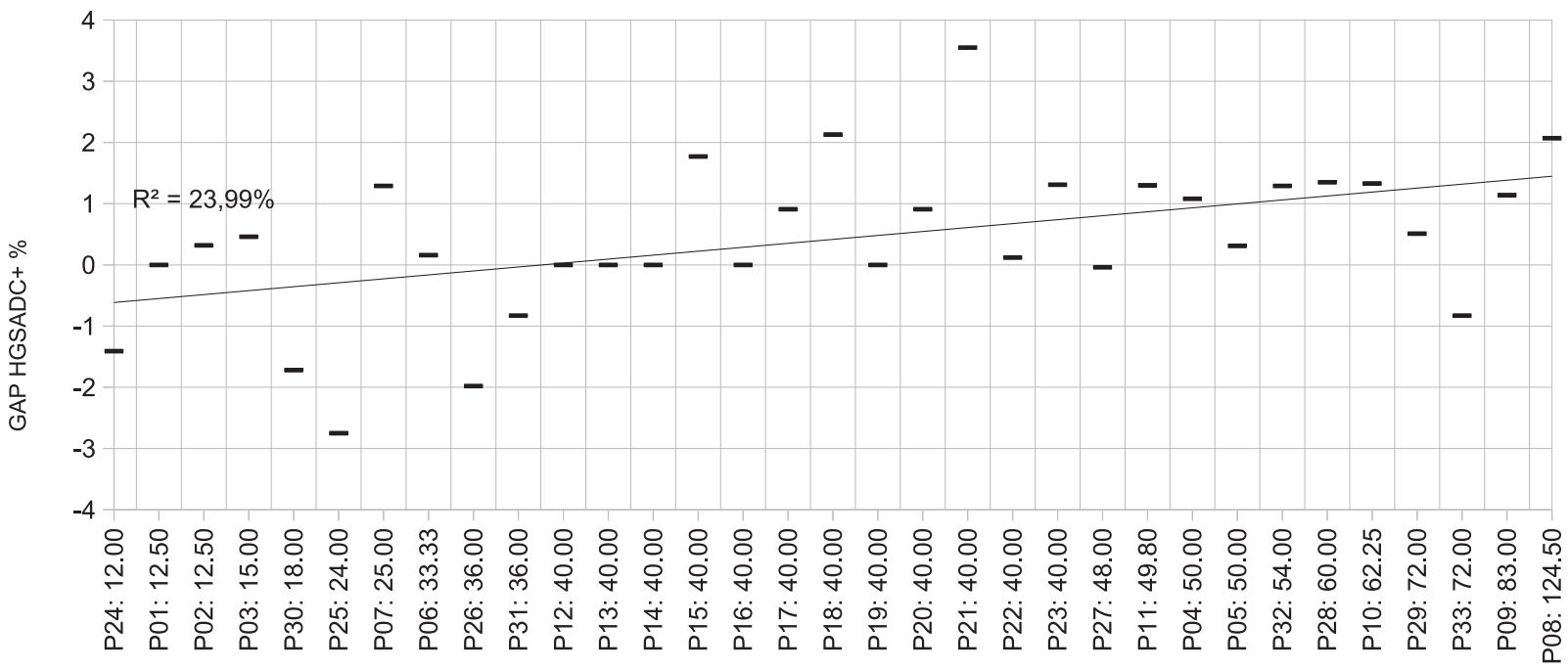

Ratio N/M

Fig. 15. Average gap based on the ratio between customers and depots.

reference. Fig. 15 shows the average gap with HGSADC+ when the instances are sorted in increasing order of the ratio between the number of customers and the number of depots. The graph presents a subtle trend suggesting that increasing the number of customers in each subproblem could make the problem harder for CoES. However, the correlation factor is $R^{2}=23.99 \%$ and needs to be further investigated.
Fig. 16 shows the average gap with HGSADC+ when the instances are sorted in increasing order of the ratio between the number of customers assigned to more than one depot and the number of depots. It measures the overlap among the subproblems. The results suggest that the performance of the coevolutionary approach is not directly affected by this characteristic, even for instances in which the overlap is important, e.g., P08 and P33. 


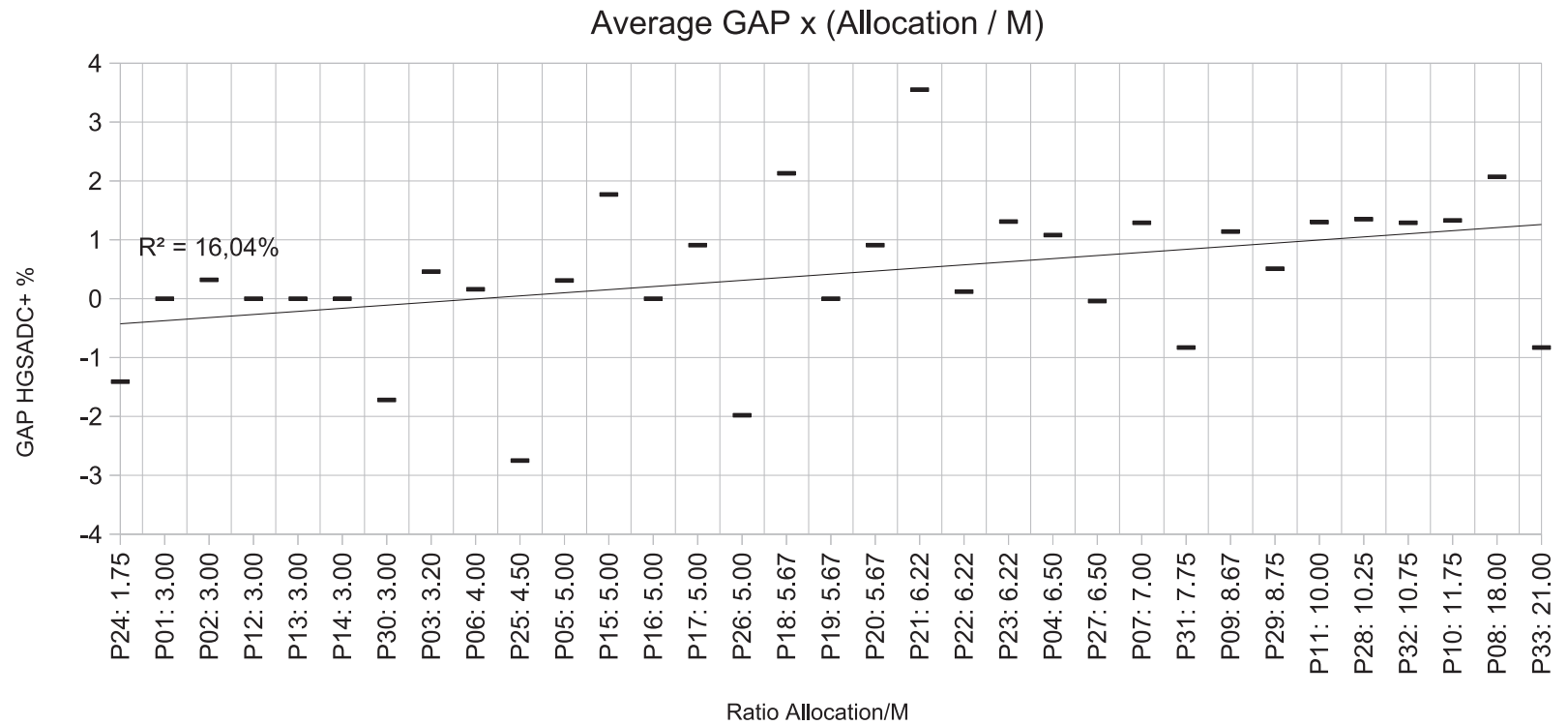

Fig. 16. Average gap based on the ratio between customer assignments and depots.

Overall, the coevolution paradigm was able to produce competitive solutions, even if its inherent parallel nature was not fully exploited in our implementation.

\subsection{Comparison of CPU times}

In order to compare the CPU times between the method proposed in this paper and HGSADC+ (Vidal et al., 2014), a conversion procedure is required. The performance of several computers is reported by Dongarra (2014) when solving dense systems of linear equations. This conversion uses the amount of floating point operations per second (flop/s) as reference and the measure is estimated using the LINPACK Benchmark (Dongarra, 2014). The processor used in Vidal et al. (2014) (AMD Opteron $2502.4 \mathrm{GHz}$ ) is reported with $1291 \mathrm{Mflop} / \mathrm{s}$. This value is computed with a matrix of order 100 (defined as problem size). However, the environment used in our work is not reported and an experiment was conducted to obtain the (flop/s) under the same conditions. The LINPACK Benchmark was obtained from Intel ${ }^{4}$ and a test was performed using 100 equations (problem size) and 100 trials. The average result of this test is $2787 \mathrm{Mflop} / \mathrm{s}$. Therefore, a scaling factor of 2.16 is obtained by dividing $2787 \mathrm{Mflop} / \mathrm{s}$ by the reported value of $1291 \mathrm{Mflop} / \mathrm{s}$ in Vidal et al. (2014).

Table 7 shows the run times of our method (in minutes) in contrast to the run times reported for HGSADC+, considering the conversion factor. The column $\mathrm{CoES}_{\text {adj }}$ corresponds to the adjusted time for our method. The average times in seconds $\mathrm{CoES}_{\text {sec }}$ reported in Table 4, column Time(s), were transformed into the corresponding adjusted values (in minutes) as follows:

$\mathrm{CoES}_{\text {adj }}(\min )=\frac{\mathrm{CoES}_{\mathrm{sec}} \times 2.16}{60}$

The column $H G S A D C+$ is the average computation time in minutes of HGSADC+. The speed-up $S$ is calculated differently depending if CoES or HGSADC+ is faster:

$S=\frac{\text { Slower }}{\text { Faster }}$

The ratio is positive when CoEs is faster. However, when HGSADC+ is faster, a minus sign is put in front of $S$ to get a negative ratio.

\footnotetext{
${ }^{4}$ Available at: http://goo.gl/1ZEYbj .
}

Table 7

Comparison of run time.

\begin{tabular}{|c|c|c|c|}
\hline \multirow[t]{2}{*}{ Inst } & \multicolumn{2}{|c|}{ Time (min) } & \multirow[t]{2}{*}{ Speedup } \\
\hline & $\mathrm{CoES}_{\mathrm{adj}}$ & HGSADC+ & \\
\hline P01 & 0.04 & 1.09 & 27.25 \\
\hline P02 & 0.02 & 1.69 & 84.50 \\
\hline P03 & 0.09 & 2.53 & 28.11 \\
\hline P04 & 6.83 & 3.43 & -1.99 \\
\hline P05 & 0.96 & 4.89 & 5.09 \\
\hline P06 & 2.78 & 3.02 & 1.09 \\
\hline P07 & 0.87 & 3.75 & 4.31 \\
\hline P08 & 28.92 & 19.93 & -1.45 \\
\hline P09 & 18.48 & 19.51 & 1.06 \\
\hline P10 & 25.92 & 17.71 & -1.46 \\
\hline P11 & 14.26 & 17.14 & 1.20 \\
\hline P12 & 0.03 & 2.85 & 95.00 \\
\hline P13 & 0.00 & 2.85 & $285.00(*)$ \\
\hline P14 & 0.00 & 2.53 & $253.00(*)$ \\
\hline P15 & 3.85 & 7.79 & 2.02 \\
\hline P16 & 0.30 & 7.75 & 25.83 \\
\hline P17 & 0.53 & 8.31 & 15.68 \\
\hline P18 & 15.45 & 14.01 & -1.10 \\
\hline P19 & 2.61 & 15.11 & 5.79 \\
\hline $\mathrm{P} 20$ & 6.85 & 16.20 & 2.36 \\
\hline $\mathrm{P} 21$ & 19.98 & 20.07 & 1.00 \\
\hline $\mathrm{P} 22$ & 7.70 & 20.00 & 2.60 \\
\hline P23 & 19.05 & 20.00 & 1.05 \\
\hline P24 & 0.00 & 2.05 & $205.00(*)$ \\
\hline $\mathrm{P} 25$ & 22.71 & 4.67 & -4.86 \\
\hline P26 & 4.45 & 8.84 & 1.99 \\
\hline $\mathrm{P} 27$ & 20.91 & 12.84 & -1.63 \\
\hline P28 & 11.56 & 19.51 & 1.69 \\
\hline P29 & 26.08 & 20.00 & -1.30 \\
\hline P30 & 0.02 & 3.04 & 152.00 \\
\hline P31 & 9.14 & 7.85 & -1.16 \\
\hline P32 & 23.49 & 15.65 & -1.50 \\
\hline P33 & 17.02 & 20.00 & 1.18 \\
\hline Average & 9.42 & 10.50 & 35.98 \\
\hline $\mathrm{S} 1$ & 7.63 & 10.09 & 36.35 \\
\hline $\mathrm{S} 2$ & 13.54 & 11.45 & 35.14 \\
\hline
\end{tabular}

Clearly, the reported computation times of CoES are generally smaller than those of HGSADC+. This computational efficiency is due to the decomposition in the coevolutionary algorithm combined with our parallel implementation (which could be improved, for example, through massive parallelism on GPUs). 


\section{Conclusion and future work}

This paper proposed a cooperative coevolutionary algorithm to solve the MDVRP. In this algorithm, each depot is associated with a population with its assigned customers. Individuals in each population represent partial single-depot solutions to the problem. Each population evolves separately, but the quality of an individual depends on its ability to cooperate with partial solutions from other populations to form a good complete solution to the MDVRP.

The results show that our coevolutionary algorithm produces competitive solutions when compared with the best known solutions, even improving some of them. Besides, it is faster than HGSDAC+, which is the best method reported in the literature. The benefit of our approach comes from its ability to decompose complex problems into simpler subproblems and evolve solutions to the subproblems in parallel. The decomposition approach also makes the method more scalable. In large MDVRP instances, it is unlikely that customers close to a depot will be allocated to a distant depot. Therefore, the coevolutionary algorithm incorporates important characteristics of a problem instance and allows a reduction of the search space. Moreover, the evolutionary engine leads to simpler representations and genetic operators. Finally, the coevolutionary algorithm proposed in this work would greatly benefit from cloud computing architectures, cluster computing and GPU programming.

One interesting avenue of research would be to integrate mathematical programming into the local searches (matheuristic). Since each subproblem reduces to a single-depot VRP, the use of mathematical programming within each population could be beneficial. Additionally, other sophisticated local search strategies could be incorporated into the EG module, like Tabu Search. Finally, we intend to improve the parallel implementation by exploiting GPU programming for the PE, CSE and EG modules.

\section{Acknowledgments}

F. B. Oliveira would like to thank the financial support from CAPES Foundation, Ministry of Education of Brazil, grant BEX 0295/14-0, for awarding the scholarship for the visit period at CIRRELT in Universite de Montréal.

R. Enayatifar and H. Javedani Sadaei would like to thank the support given by the Brazilian Agency CAPES.

F. G. Guimarães would like to thank the support given by the National Council for Scientific and Technological Development (CNPq grant no. 312276/2013-3), Brazil.

J.-Y. Potvin would like to thank the Natural Sciences and Engineering Research Council of Canada (NSERC) for its financial support.

\section{References}

Aote, S., Raghuwanshi, M., \& Malik, L. (2015). A new particle swarm optimizer with cooperative coevolution for large scale optimization. In S. C. Satapathy, B. N. Biswal, S. K. Udgata, \& J. Mandal (Eds.), Proceedings of the third international conference on frontiers of intelligent computing: Theory and applications (FICTA) 2014. In Advances in intelligent systems and computing: vol. 327 (pp. 781-789). Springer International Publishing. doi:10.1007/978-3-319-11933-5_88.

Baldacci, R., \& Mingozzi, A. (2009). A unified exact method for solving different classes of vehicle routing problems. Mathematical Programming, 120(2), 347-380. doi:10.1007/s10107-008-0218-9.

Blecic, I., Cecchini, A., \& Trunfio, G. A. (2014). Fast and accurate optimization of a GPU-accelerated $\{\mathrm{CA}\}$ urban model through cooperative coevolutionary particle swarms. Procedia Computer Science, 29(0), 1631-1643. 2014 International conference on computational science http://dx.doi.org/10.1016/j.procs.2014.05.148.

Bodin, L., Golden, B., Assad, A., \& Ball, M. (1983). Routing and scheduling of vehicles and crews: The state of the art. Computers \&' Operations Research, 10(2), 63-211.
Campelo, F. (2015). Lecture notes on design and analysis of experiments. Version 2.11, creative commons BY-NC-SA 4.0. https://github.com/fcampelo/Design-andAnalysis-of-Experiments.

Chen, H., Mori, Y., \& Matsuba, I. (2014). Solving the balance problem of massively multiplayer online role-playing games using coevolutionary programming. Applied Soft Computing, 18(0), 1-11. http://dx.doi.org/10.1016/j.asoc.2014.01.011.

Chen, H., Zhu, Y., \& Hu, K. (2010). Discrete and continuous optimization based on multiswarm coevolution. Natural Computing, 9(3), 659-682. doi:10.1007/s11047-0099174-4.

Chen, P., \& Xu, X. (2008). A hybrid algorithm for multi-depot vehicle routing problem. In IEEE international conference on service operations and logistics, and informatics, 2008. IEEE/SOLI 2008: vol. 2 (pp. 2031-2034). doi:10.1109/SOLI.2008.4682866.

Cordeau, J.-F., Gendreau, M., \& Laporte, G. (1997). A tabu search heuristic for periodic and multi-depot vehicle routing problems. Networks, 30(2), 105-119.

Cordeau, J.-F., \& Maischberger, M. (2012). A parallel iterated tabu search heuristic for vehicle routing problems. Computers E'Operations Research, 39(9), 2033-2050. http://dx.doi.org/10.1016/j.cor.2011.09.021.

Core TeamR. (2015). R: A language and environment for statistical computing. Vienna, Austria: R Foundation for Statistical Computing.

Doerner, K., \& Schmid, V. (2010). Survey: Matheuristics for rich vehicle routing problems. In M. Blesa, C. Blum, G. Raidl, A. Roli, \& M. Sampels (Eds.), Hybrid metaheuristics. In Lecture notes in computer science: vol. 6373 (pp. 206-221). Berlin, Heidelberg: Springer. doi:10.1007/978-3-642-16054-7_15.

Dongarra, J. J. (2014). Performance of various computers using standard linear equations software (Linpack benchmark report). Technical report, CS-89-85.University of Tennessee Computer Science.

Ebner, M. (2006). Coevolution and the red queen effect shape virtual plants. Genetic Programming and Evolvable Machines, 7, 103-123. doi:10.1007/s10710-006-7013-2. Engelbrecht, A. (2007). Computational intelligence: An introduction. Wiley.

Escobar, J., Linfati, R., Toth, P., \& Baldoquin, M. (2014). A hybrid granular tabu search algorithm for the multi-depot vehicle routing problem. Journal of Heuristics, 20(5), 483-509. doi:10.1007/s10732-014-9247-0.

Ficici, S. G. (2004). Solution concepts in coevolutionary algorithms. Waltham, MA, USA: (Ph.D. thesis). Faculty of Brandeis University.AAI3127125

Freitas, A. R. R., Guimarães, F. G., Pedrosa Silva, R. C., \& Souza, M. J. F. (2014). Memetic self-adaptive evolution strategies applied to the maximum diversity problem. $O p$ timization Letters, 8(2), 705-714. doi:10.1007/s11590-013-0610-0.

Gendreau, M., Potvin, J.-Y., Bräumlaysy, O., Hasle, G., \& Løkketangen, A. (2008). Metaheuristics for the vehicle routing problem and its extensions: A categorized bibliography. In B. Golden, S. Raghavan, \& E. Wasil (Eds.), The vehicle routing problem: Latest advances and new challenges. In Operations research/computer science interfaces: vol. 43 (pp. 143-169). US: Springer. doi:10.1007/978-0-387-77778-8 7.

Gu, J., Gu, M., Cao, C., \& Gu, X. (2010). A novel competitive co-evolutionary quantum genetic algorithm for stochastic job shop scheduling problem. Computers E'Operations Research, 37(5), 927-937. Disruption management http://dx.doi.org/10.1016/ j.cor.2009.07.002.

Ho, W., Ho, G. T., Ji, P., \& Lau, H. C. (2008). A hybrid genetic algorithm for the multidepot vehicle routing problem. Engineering Applications of Artificial Intelligence, 21(4), 548-557. http://dx.doi.org/10.1016/j.engappai.2007.06.001.

Jansen, T., \& Wiegand, R. (2003). Sequential versus parallel cooperative coevolutionary (1+1) EAS. In The 2003 congress on evolutionary computation, 2003. CEC '03: vol. 1 (pp. 30-37) doi:10.1109/CEC.2003.1299553.

Jansen, T., \& Wiegand, R. P. (2004). The cooperative coevolutionary (1+1) EA. Evolutionary Computation, 12(4), 405-434.

Karakatič, S., \& Podgorelec, V. (2015). A survey of genetic algorithms for solving multi depot vehicle routing problem. Applied Soft Computing, 27(0), 519-532. http://dx.doi.org/10.1016/j.asoc.2014.11.005.

Katada, Y., \& Handa, Y. (2010). Tracking the red queen effect by estimating features of competitive co-evolutionary fitness landscapes. In 2010 IEEE congress on evolutionary computation (CEC) (pp. 1-8). doi:10.1109/CEC.2010.5586188.

Ladjici, A., \& Boudour, M. (2011). Nash-Cournot equilibrium of a deregulated electricity market using competitive coevolutionary algorithms. Electric Power Systems Research, 81(4), 958-966. http://dx.doi.org/10.1016/j.epsr.2010.11.016.

Ladjici, A., Tiguercha, A., \& Boudour, M. (2014). Nash equilibrium in a twosettlement electricity market using competitive coevolutionary algorithms. International Journal of Electrical Power \& Energy Systems, 57(0), 148-155. http://dx.doi.org/10.1016/j.ijepes.2013.11.045.

Lau, H., Chan, T., Tsui, W., \& Pang, W. (2010). Application of genetic algorithms to solve the multidepot vehicle routing problem. IEEE Transactions on Automation Science and Engineering, 7(2), 383-392. doi:10.1109/TASE.2009.2019265.

Li, M., Guimarães, F., \& Lowther, D. A. (2015). Competitive co-evolutionary algorithm for constrained robust design. IET Science, Measurement \& Technology, 9(2), 218-223. doi:10.1049/iet-smt.2014.0204.

Li, X., \& Yao, X. (2012). Cooperatively coevolving particle swarms for large scale optimization. IEEE Transactions on Evolutionary Computation, 16(2), 210-224. doi:10.1109/TEVC.2011.2112662.

Liu, C.-Y. (2013). An improved adaptive genetic algorithm for the multi-depot vehicle routing problem with time window. Journal of Networks, 8(5), 1035-1042.

Luke, S. (2013). Essentials of metaheuristics (2nd). Lulu. Available for free at http://cs.gmu.edu/ sean/book/metaheuristics/

Maischberger, M., \& Cordeau, J.-F. (2011). Solving variants of the vehicle routing problem with a simple parallel iterated tabu search. In J. Pahl, T. Reiners, \& S. Voß (Eds.), Network optimization. In Lecture notes in computer science: vol. 6701 (pp. 395-400). Berlin and Heidelberg: Springer. doi:10.1007/978-3-642-21527-8_44. 
130

F.B. de Oliveira et al./ Expert Systems With Applications 43 (2016) 117-130

Mi, Y., Li, X., \& Yo, X. (2014). Cooperative coevolution with route distance grouping for large-scale capacitated arc routing problems. IEEE Transactions on Evolutionary Computation, 18(3), 435-449. doi:10.1109/TEVC.2013.2281503.

Montgomery, D., \& Ringer, G. (2011). Applied statistics and probability for engineers (Fth). John Wiley \& Sons.

Montoya-Torres, J. R., Franco, J. L., Isaza, S. N., Jiménez, H. F., \& HerazoPadilla, N. (2015). A literature review on the vehicle routing problem with ultiple depots. Computers \& Industrial Engineering, 79(0), 115-129. http://dx.doi.org/ 10.1016/j.cie.2014.10.029.

Pisinger, D., \& Ropke, S. (2007). A general heuristic for vehicle routing problems. Computers \& Operations Research, 34(8), 2403-2435. http://dx.doi.org/ 10.1016/j.cor.2005.09.012.

Popovici, E., \& De Jong, K. (2006). Sequential versus parallel cooperative coevolutionary algorithms for optimization. In IEEE congress on evolutionary computation, 2006. CE 2006 (pp. 1610-1617). doi:10.1109/CEC.2006.1688501.

Potvin, J.-Y. (2009). State-of-the art review-evolutionary algorithms for vehicle routing. INFORMS Journal on Computing, 21(4), 518-548. doi:10.1287/ijoc.1080.0312.

Prins, C. (2004). A simple and effective evolutionary algorithm for the vehile routing problem. Computers \& Operations Research, 31(12), 1985-2002. http://dx.doi.org/10.1016/S0305-0548(03)00158-8.

Renaud, J., Laporte, G., \& Boctor, F. F. (1996). A tabu search heuristic for the multidepot vehicle routing problem. Computers E Operations Research, 23(3), 229-235. http://dx.doi.org/10.1016/0305-0548(95)00026-P.

Shang, R., Wang, Y., Wang, J., Jiao, L., Wang, S., \& Qi, L. (2014). A multi-population cooperative coevolutionary algorithm for multi-objective capacitated arc routing problem. Information Sciences, 277, 609-642. http://dx.doi.org/10.1016/j. ins.2014.03.008.

Siqueira Ruela, A., Gadelha Guimaraes, F., Rabelo Oliveira, R., Neves, B., Peixoto Amorim, V., \& Fraga, L. (2013). A parallel hybrid genetic algorithm on cloud computting for the vehicle routing problem with time windows. In 2013 IEEE international conference on systems, man, and cybernetics (SMC) (pp. 2467-2472). doi:10.1109/SMC.2013.421.
Subramanian, A., Uchoa, E., \& Ochi, L. S. (2013). A hybrid algorithm for a class of vehicle routing problems. Computers E' Operations Research, 40(10), 2519-2531. http://dx.doi.org/10.1016/j.cor.2013.01.013.

Vidal, T., Crainic, T. G., Gendreau, M., Lahrichi, N., \& Reid, W. (2012). A hybrid genetic algorithm for multidepot and periodic vehicle routing problems. Operations Research, 60(3), 611-624. doi:10.1287/opre.1120.1048.

Vidal, T., Crainic, T. G., Gendreau, M., \& Prins, C. (2014). Implicit depot assignments and rotations in vehicle routing heuristics. European Journal of Operational Research, 237(1), 15-28. http://dx.doi.org/10.1016/j.ejor.2013.12.044.

Wang, H.-F., \& Chen, Y.-Y. (2013a). A coevolutionary algorithm for the flexible delivery and pickup problem with time windows. International Journal of Production Economics, 141(1), 4-13. Meta-heuristics for manufacturing scheduling and logistics problems http://dx.doi.org/10.1016/j.ijpe.2012.04.011.

Wang, H.-F., \& Chen, Y.-Y. (2013b). A coevolutionary algorithm for the flexible delivery and pickup problem with time windows. International Journal of Production Economics, 141(1), 4-13. Meta-heuristics for manufacturing scheduling and logistics problems http://dx.doi.org/10.1016/j.ijpe.2012.04.011.

Wang, X., Chen, H., \& Huang, M. (2014). QoS multicast routing protocol oriented to cognitive network using competitive coevolutionary algorithm. Expert Systems with Applications, 41(10), 4513-4528. http://dx.doi.org/10.1016/j.eswa.2014.01. 020.

Wenjing, Z., \& Ye, J. (2010). An improved particle swarm optimization for the multidepot vehicle routing problem. In 2010 International conference on E-business and e-government (ICEE) (pp. 3188-3192). doi:10.1109/ICEE.2010.803.

Yo, B., Yang, Z. Z., \& Xis, J.-X. (2011). A parallel improved ant colony optimization for multi-depot vehicle routing problem. Journal of the Operational Research Society, 62(1), 183-188. 